\title{
STRICTLY ANALYTIC FUNCTIONS ON $p$-ADIC ANALYTIC OPEN SETS
}

\author{
Kamal Boussaf
}

\begin{abstract}
Let $K$ be an algebraically closed complete ultrametric field. M. Krasner and P. Robba defined theories of analytic functions in $K$, but when $K$ is not spherically complete both theories have the disadvantage of containing functions that may not be expanded in Taylor series in some disks. On other hand, affinoid theories are only defined in a small class of sets (union of affinoid sets) [2], [13] and [17]. Here, we suppose the field $K$ topologically separable (example $\mathbb{C}_{p}$ ). Then, we give a new definition of strictly analytic functions over a large class of domains called analoid sets. Our theory uses the notion of $T$-sequence which caracterizes analytic sets in the sense of Robba. Thereby we obtain analytic functions satisfying the property of analytic continuation and which, however, will admit expansion in power series (resp. Laurent series) in any disk (resp. in any annulus). Moreover, the algebra of analytic functions will be stable by derivation. The process consists of defining a large class of analytic sets $D$, and a class of admissible sets making a covering of such a $D$, so that we obtain a sheaf on $D$. We finally give an example of differential equation whose solutions are strictly analytic functions in an analoid set. Such an example might not be involved in theories based on affinoid sets.
\end{abstract}

\section{Preliminaries}

Let $K$ be an algebraically closed field complete for an ultrametric absolute value. We recall some standard notations and definitions.

Given $a \in K$ and $r>0, d(a, r)$ (resp. $d\left(a, r^{-}\right)$, resp. $\left.C(a, r)\right)$ denotes the circumferenced disk $\{x \in K|| x-a \mid \leq r\}$ (resp. the noncircumferenced disk $\{x \in K|| x-a \mid<r\}$, resp. the circle $\{x \in$ $K|| x-a \mid=r\})$. We call $a$ class of $d(a, r)$ any non-circumferenced disk $d\left(b, r^{-}\right)$with $|a-b| \leq r$.

Let $r^{\prime}>0$ and $r^{\prime \prime}>r^{\prime}, \Gamma\left(a, r^{\prime}, r^{\prime \prime}\right)\left(\right.$ resp. $\left.\Delta\left(a, r^{\prime}, r^{\prime \prime}\right)\right)$ denotes the annulus $\left\{x \in K\left|r^{\prime}<\right| x-a \mid<r^{\prime \prime}\right\}$ (resp. $\left\{x \in K\left|r^{\prime} \leq\right| x-a \mid \leq r^{\prime \prime}\right\}$ ). 
Let $D$ be an infinite subset of $K$. Then $R(D)$ denotes the set of rational functions $h \in K(x)$ with no poles in $D$. This is a $K$-subalgebra of the algebra $K^{D}$ of all functions from $D$ into $K$. Then $R(D)$ is provided with the topology $\mathcal{U}_{D}$ of uniform convergence on $D$, and is a topological group for this topology. $H(D)$ denotes the completion of $R(D)$ for this topology and its elements are named the analytic elements on $D[\mathbf{4}],[\mathbf{6}]$, [11].

Definition. A set $D$ is said to be analytic if for every $f \in H(D)$ for every $a \in D$ and $r>0$, the property $f(x)=0$ whenever $x \in d(a, r) \cap D$ implies $f(x)=0$ whenever $x \in D$.

In Theorem 0 we recall the characterization of analytic sets by using $T$-sequences.

Example and remarks. Let $\left(a_{n}\right)_{n \in \mathbb{N}}$ be a sequence in $K$ such that $\left|a_{n}\right|<\left|a_{n+1}\right| \forall n \in \mathbb{N}, \lim _{n \rightarrow \infty}\left|a_{n}\right|=1$ and $\sum_{n=0}^{\infty}-\log \left|a_{n}\right|<+\infty$. Let $\rho \in] 0,1\left[\right.$ and $\left(r_{n}\right)_{n \in \mathbb{N}}$ be a sequence in $] 0, \rho[$. Then by [6, Proposition 36.5], the open set $D=d(0,2) \backslash\left(\bigcup_{n \in \mathbb{N}} d\left(a_{n}, r_{n}{ }^{-}\right)\right)$is analytic.

Notice that Robba's definition of analytic functions may extend to open sets like $D$. However, in a not spherically complete field such as $\mathbb{C}_{p}$, this definition gives functions that may not be expanded in Taylor series in some disks.

On the other hand, affinoid sets (resp. "connected" affinoid sets i.e.: infraconnected affinoid sets) are defined by Fresnel and Van Der Put in [7] and were used to construct a theory of analytic functions defined by a sheaf of analytic elements on affinoid sets. But, for example, if we consider the analytic set $D$ defined above, we see that such a set can't be covered by an increasing sequence of infraconnected affinoid sets. Therefore, by Fresnel and Van Der Put process, one obtains, for example, the characteristic function of the set $d(0,2) \backslash d\left(0,1^{-}\right)$as an analytic function on $D([\mathbf{7}, 1.7])$. Hence, we see that the family of affinoid sets is too small to give a general theory of analytic functions, satisfying the principle of analytic continuation.

The aim here is to construct a large family of analytic functions, rich in properties, and defined on a large class of open analytic sets named analoid sets, as are Robba's sets, but avoiding the inconvenience of containing functions that may not be expanded in Taylor series in some disks in a non-spherically complete field. Particularly, we will see that 
strictly analytic functions satisfy the principle of analytic continuation on any analoid set (Theorem 28).

Next, we recall some preliminaries definitions and particularly this of $T$-sequences.

A sequence $\left(a_{n}\right)_{n \in \mathbb{N}}$ in $K$ is said to be an increasing distances sequence (resp. a decreasing distances sequence) if the sequence $\left|a_{n+1}-a_{n}\right|$ is strictly increasing (resp. decreasing) and has a limit $l \in \mathbb{R}^{*}{ }_{+}$.

A sequence $\left(a_{n}\right)_{n \in \mathbb{N}}$ is said to be a monotonous distances sequence if it is either an increasing distances sequence or a decreasing distances sequence.

A sequence $\left(a_{n}\right)_{n \in \mathbb{N}}$ in $K$ is said to be an equal distances sequence if $\left|a_{n}-a_{m}\right|=\left|a_{m}-a_{q}\right|$ whenever $n, m, q \in \mathbb{N}$ such that $n \neq m \neq q$.

A set $D$ in $K$ is said to be infraconnected if for every $a \in D$, the mapping $I_{a}$ from $D$ to $\mathbb{R}_{+}$defined by $I_{a}(x)=|x-a|$ has an image whose closure in $\mathbb{R}_{+}$is an interval. (In other words, a set $D$ is not infraconnected if and only if there exist $a$ and $b \in D$ and an annulus $\Gamma\left(a, r_{1}, r_{2}\right)$ with $0<r_{1}<r_{2}<|a-b|$ such that $\Gamma\left(a, r_{1}, r_{2}\right) \cap D=\emptyset$. $)$

As usual, given a set $A$ in $K$ and a point $a \in K$, we denote by $\delta(a, A)$ the distance from $a$ to $A$.

Let $D$ be an infinite set in $K$, and let $a \in D$. If $D$ is bounded of diameter $r$, we denote by $\widetilde{D}$ the disk $d(a, r)$, and if $D$ is not bounded, we put $\widetilde{D}=K$. Then, $\widetilde{D} \backslash \bar{D}$ is known to admit a partition of the form $\left(d\left(a_{i}, r_{i}^{-}\right)_{i \in J}\right)$, with $r_{i}=\delta\left(a_{i}, D\right)$ for each $i \in J$. The disks $d\left(a_{i}, r_{i}^{-}\right)_{i \in J}$, are named the holes of $D$.

\section{Monotonous filters.}

Let $a \in \widetilde{D}$ and $S \in \mathbb{R}_{+}^{*}$ be such that $\Gamma(a, r, S) \cap D \neq \emptyset$ whenever $r \in] 0, S[$ (resp. $\Gamma(a, S, r) \cap D \neq \emptyset$ whenever $r>S$ ). We call an increasing (resp. a decreasing) filter of center a and diameter $S$, on $D$ the filter $\mathcal{F}$ on $D$ that admits for base the family of sets $\Gamma(a, r, S) \cap D(\operatorname{resp} . \Gamma(a, S, r) \cap$ $D)$. For every sequence $\left(r_{n}\right)_{n \in \mathbb{N}}$ such that $r_{n}<r_{n+1}$ (resp. $r_{n}>$ $\left.r_{n+1}\right)$ and $\lim _{n \rightarrow \infty} r_{n}=S$, it is seen that the sequence $\Gamma\left(a, r_{n}, S\right) \cap D$ (resp. $\left.\Gamma\left(a, S, r_{n}\right) \cap D\right)$ is a base of $\mathcal{F}$ and such a base is called a canonical base $[\mathbf{6}]$.

Given an increasing (resp. a decreasing) filter $\mathcal{F}$ on $D$ of center $a$ and diameter $r$, we will denote by $\mathcal{P}_{D}(\mathcal{F})$ the set $\{x \in D|| x-a \mid \geq r\}$ (resp. the set $\{x \in D|| x-a \mid \leq r\}$ ). Further $\mathcal{P}_{D}(\mathcal{F})$ will be named the D-beach of $\mathcal{F}$. 
We call a monotonous filter on $D$ a filter which is either an increasing filter or a decreasing filter.

Given a monotonous filter $\mathcal{F}$ we will denote by $\operatorname{diam}(\mathcal{F})$ its diameter.

The field $K$ is said to be spherically complete if each nested sequence of disks has a nonempty intersection. The field $\mathbb{C}_{p}$, for example, is not spherically complete. However, every algebraically closed complete ultrametric field admits a spherically complete algebraically closed extension $[6]$.

Let $\mathcal{F}$ be an increasing (resp. a decreasing) filter of center $a$ and diameter $S$ on $D$. The filter $\mathcal{F}$ is said to be pierced if for every $r \in] 0, S[$, (resp. $r>S), \Gamma(a, r, S)(\operatorname{resp} . \Gamma(a, S, r))$ contains some hole $T_{m}$ of $D$.

\section{Monotonous distances holes sequences.}

Let $a \in \widetilde{D}$. Let $\left(T_{m, i}\right)_{\substack{1 \leq i \leq s(m) \\ m \in \mathbb{N}}}$ be a sequence of holes of $D$ which satisfies $\delta\left(a, T_{m, i}\right)=d_{m}(1 \leq i \leq s(m), m \in \mathbb{N}), d_{m}<d_{m+1}\left(\right.$ resp. $d_{m}>$ $\left.d_{m+1}\right)$, and $\lim _{m \rightarrow \infty} d_{m}=R>0$.

The sequence $\left(T_{m, i}\right)_{\substack{1 \leq i \leq s(m) \\ m \in \mathbb{N}}}$ is called an increasing (resp. decreasing) distances holes sequence that runs the increasing (resp. decreasing) filter $\mathcal{F}$ of center a, of diameter $R$. The filter $\mathcal{F}$ will be named the increasing (resp. decreasing) filter associated to the sequence $\left(T_{m, i}\right)_{\substack{1 \leq i \leq s(m) \\ m \in \mathbb{N}}}$. The $D$-beach of $\mathcal{F}$ will be also named the D-beach of $\left(T_{m, i}\right)_{\substack{1 \leq i \leq s(m) \\ m \in \mathbb{N}}}$.

Finally, an increasing (resp. decreasing) distances holes sequence will be called a monotonous distances holes sequence and the sequence $\left(d_{m}\right)_{m \in \mathbb{N}}$ is called the monotony of the monotonous distances holes sequence.

Let $\left(T_{m, i}\right)_{\substack{1 \leq i \leq s(m) \\ m \in \mathbb{N}}}$ be a monotonous distances holes sequence and for every $(m, i)(i \in\{1, \ldots, s(m)\}, m \in \mathbb{N})$, let $\rho_{m, i}=\operatorname{diam}\left(T_{m, i}\right)$. The number $\liminf _{m \rightarrow \infty}\left(\min _{1 \leq i \leq s(m)}\left(\rho_{m, i}\right)\right)$ (resp. $\left.\limsup _{m \rightarrow \infty}\left(\max _{1 \leq i \leq s(m)}\left(\rho_{m, i}\right)\right)\right)$ will be called inferior limit-piercing (resp. superior limit-piercing) of the sequence $\left(T_{m, i}\right)_{\substack{1 \leq i \leq s(m) \\ m \in \mathbb{N}}}$.

If a monotonous holes sequence of diameter $r$ has an inferior limitpiercing $\rho>0$ and a superior limit-piercing $\rho^{\prime}<r$, it will be said correctly pierced.

A set $D$ will be said to be correctly pierced if every monotonous distances holes sequence of $D$ with a not empty $D$-beach is correctly pierced. 
A set $D$ is said to be well pierced if $\delta(\bar{D}, K \backslash \bar{D})>0$, i.e. the set of diameters of holes of $D$ has a strictly positive lower bound.

\section{Weighted sequences.}

We call a weighted sequence a sequence $\left(T_{m, i}, q_{m, i}\right)_{\substack{1 \leq i \leq s(m) \\ m \in \mathbb{N}}}$ with $\left(T_{m, i}\right)_{\substack{1 \leq i \leq s(m) \\ m \in \mathbb{N}}}$ a monotonous distances holes sequence and $\left(q_{m, i}\right)_{\substack{1 \leq i \leq s(m) \\ m \in \mathbb{N}}}$ a sequence of nonnegative integers. Given $m \in \mathbb{N}$ and $i \in\{1, \ldots, s(m)\}$, $q_{m, i}$ is called the weight of $T_{m, i}$. The $D$-beach of $\left(T_{m, i}\right)_{\substack{1 \leq i \leq s(m) \\ m \in \mathbb{N}}}$ is also named the D-beach of $\left(T_{m, i}, q_{m, i}\right)_{\substack{1 \leq i \leq s(m) \\ m \in \mathbb{N}}}$.

The monotonous filter associated to $\left(T_{m, i}\right)_{\substack{1 \leq i \leq s(m) \\ m \in \mathbb{N}}}$, is also called filter associated to $\left(T_{m, i}, q_{m, i}\right)_{\substack{1 \leq i \leq s(m) \\ m \in \mathbb{N}}}$.

A weighted sequence is said to be correctly pierced if its associated monotonous distances holes sequence is.

For every $m \in \mathbb{N}$, we put

$\Omega_{m}=\max _{1 \leq i \leq s(m)}\left(q_{m, i} \log \left(\frac{d_{m}}{\rho_{m, i}}\right)+\sum_{\substack{j \neq i \\ 1 \leq j \leq s(m)}} q_{m, j}\left(\log d_{m}-\log \left|a_{m, j}-a_{m, i}\right|\right)\right)$

The sequence $\left(\Omega_{m}\right)_{m \in \mathbb{N}}$ will be called perturbations sequence of the weighted sequence.

We will say that the weighted sequence has a perturbations sequence bounded by $\lambda \in \mathbb{R}_{+}$, if $\sup \Omega_{m} \leq \lambda$. More generally we say that the weighted sequence has a bounded perturbations sequence, if $\sup _{m \in \mathbb{N}} \Omega_{m}<+\infty$.

A weighted sequence $\left(T_{m, i}, q_{m, i}\right)_{1 \leq i \leq s(m)}$ will be said to be idempotent if $q_{m, i}=0$ or 1 for all $(m, i),(1 \leq i \leq s(m), m \in \mathbb{N})$ and $q_{m, i} \neq 0$ for infinitely many $(m, i)$.

Let $\mathcal{S}_{1}$ and $\mathcal{S}_{2}$ be two monotonous distances holes (resp. weighted) sequence. We will say that $\mathcal{S}_{1}$ and $\mathcal{S}_{2}$ are cofiltring if they are associated to the same monotonous filter of $K$.

We see that two cofiltring sequences have the same centers and the same $D$-beach. 
Let $\mathcal{S}^{\prime}=\left(T_{m, i}^{\prime}\right)_{\substack{1 \leq i \leq k^{\prime}(m) \\ m \in \mathbb{N}}}$ and $\mathcal{S}^{\prime \prime}=\left(T_{m, i}^{\prime \prime}\right)_{\substack{1 \leq i \leq k^{\prime \prime}(m) \\ m \in \mathbb{N}}}$ be two cofiltring monotonous distances holes sequences, of center $a$. The holes of the set

$$
D^{\prime}=K \backslash\left(\left(\cup_{\substack{1 \leq i \leq k^{\prime}(m) \\ m \in \mathbb{N}}} T_{m, i}^{\prime}\right) \cup\left(\cup_{\substack{1 \leq i \leq k^{\prime \prime}(m) \\ m \in \mathbb{N}}} T_{m, i}^{\prime \prime}\right)\right)
$$

form a monotonous distances sequence which is cofiltring to $\mathcal{S}^{\prime}$ and $\mathcal{S}^{\prime \prime}$. We will denote it by $\mathcal{S}^{\prime} \cup \mathcal{S}^{\prime \prime}$. Thus, $\mathcal{S}^{\prime} \cup \mathcal{S}^{\prime \prime}$ is in the form $\left(T_{m, i}\right)_{\substack{1 \leq i \leq k(m) \\ m \in \mathbb{N}}}$, and we remark that a hole of $\mathcal{S}^{\prime} \cup \mathcal{S}^{\prime \prime}$ is either a hole of $\mathcal{S}^{\prime}$ or a hole of $\mathcal{S}^{\prime \prime}$.

\section{4. $T$-sequences.}

Let $\mathcal{S}=\left(T_{m, i}, q_{m, i}\right)_{\substack{1 \leq i \leq s(m) \\ m \in \mathbb{N}}}$ be an increasing (resp. decreasing) weighted sequence and for all $m \in \mathbb{N}$, let $q_{m}=\sum_{i=1}^{s(m)} q_{m, i}$. The weighted sequence $\mathcal{S}$ will be said to be a $T$-sequence if it satisfies:

$\lim _{m \rightarrow \infty}\left(\sup _{1 \leq j \leq s(m)}\left[\left(\frac{d_{m}}{\rho_{m, j}}\right)^{q_{m, j}} \prod_{\substack{i \neq j \\ 1 \leq j \leq s(m)}}\left(\frac{d_{m}}{\left|a_{m, i}-a_{m, j}\right|}\right)^{q_{m, i}}\right] \prod_{n=1}^{m-1}\left(\frac{d_{n}}{d_{m}}\right)^{q_{n}}\right)=0$

(resp.

$\left.\lim _{m \rightarrow \infty}\left(\sup _{1 \leq j \leq s(m)}\left[\left(\frac{d_{m}}{\rho_{m, j}}\right)^{q_{m, j}} \prod_{\substack{i \neq j \\ 1 \leq j \leq s(m)}}\left(\frac{d_{m}}{\left|a_{m, i}-a_{m, j}\right|}\right)^{q_{m, i}}\right] \prod_{n=1}^{m-1}\left(\frac{d_{m}}{d_{n}}\right)^{q_{n}}\right)=0\right)$.

Remark. A weighted sequence $\left(T_{m, i}, q_{m, i}\right)_{\substack{1 \leq i \leq s(m) \\ m \in \mathbb{N}}}$ is a $T$-sequence if and only if

$$
\begin{aligned}
& \lim _{m \rightarrow \infty}\left(-\sup _{1 \leq j \leq s(m)}\left[q_{m, j}\left(\log d_{m}-\log \rho_{m, j}\right)\right.\right. \\
&\left.\left.+\sum_{\substack{i \neq j \\
1 \leq i \leq s(m)}} q_{m, i}\left(\log d_{m}-\log \left|a_{m, i}-a_{m, j}\right|\right)\right]+\sum_{n=1}^{m-1} q_{n}\left|\log d_{m}-\log d_{n}\right|\right)=+\infty .
\end{aligned}
$$


Theorem 0. Let $D$ be a set in $K$. Then $D$ is analytic if and only if any $T$-sequence of $D$ has an empty $D$-beach.

Proof: By [6, Theorem 38.8], we know that $D$ is analytic if and only if $D$ is infraconnected and any $T$-sequence of holes of $D$ has an empty $D$-beach. Thus we only have to show that if $D$ is not infraconnected, then $D$ admits a $T$-sequence with a not empty $D$-beach. Let us suppose that $D$ is not infraconnected. Then there exist $a, b \in D$ and $r_{1}, r_{2} \in \mathbb{R}_{+}$ such that $0<r_{1}<r_{2}<|a-b|$ and $\Gamma\left(a, r_{1}, r_{2}\right) \cap D=\emptyset$. Hence, we see that every element of $\Gamma\left(a, r_{1}, r_{2}\right)$ belongs to a hole of $D$. So, it is easly seen that $\Gamma\left(a, r_{1}, r_{2}\right)$ admits a partition $\mathcal{P}$ by a family of holes of $D$. By $[\mathbf{1 2}$, Propositions 1.2 and 2.5] there exist $t \in] 0, r_{2}\left[, u \in d\left(a, r_{2}\right)\right.$ and an increasing idempotent $T$-sequence $\mathcal{S}$ with holes in $\mathcal{P}$, of center $u$ and diameter $t$. Then since $|b-u| \geq t$, we see that $\mathcal{S}$ is a $T$-sequence of holes of $D$ with a not empty $D$-beach.

\section{Strictly analytic functions}

\section{Polar and $T$-polar sequences.}

Let $D$ be infraconnected, let $a \in K$, let $r>0$ and let $\rho \in] 0, r[$. We call an increasing (resp. decreasing) polar sequence, of center a, of diameter $r$ and separation $\rho$ every sequence of the form $\left(b_{m, i}\right)_{\substack{1 \leq i \leq k(m) \\ m \in \mathbb{N}}}$

satisfying: $b_{m, i} \in \widetilde{D} \backslash D, \forall(m, i)(m \in \mathbb{N}, i \in\{1, \ldots, k(m)\})$, with $\left|b_{m, i}-a\right|=\left|b_{m, j}-a\right|=d_{m}$ whenever $i, j \in\{1, \ldots, k(m)\},\left|b_{m, i}-a\right|<$ $\left|b_{m+1, j}-a\right|$ (resp. $\left.\left|b_{m, i}-a\right|>\left|b_{m+1, j}-a\right|\right)$ whenever $1 \leq i \leq k(m)$ and $1 \leq j \leq k(m+1), \lim _{m \rightarrow \infty} d_{m}=r$ and $\inf _{(m, i) \neq(n, j)}\left|b_{m, i}-b_{n, j}\right|=\rho$. The sequence $\left(d_{m}\right)_{m \in \mathbb{N}}$ is called the monotony of the polar sequence. We call the D-beach of $\left(b_{m, i}\right)_{\substack{1 \leq i \leq k(m) \\ m \in \mathbb{N}}}$ the set $D \cap\left(K \backslash d\left(a, r^{-}\right)\right)$(resp. $\left.D \cap d(a, r)\right)$.

A polar sequence $\left(b_{m, i}\right)_{\substack{1 \leq i \leq k(m) \\ m \in \mathbb{N}}}$ is called $T$-polar sequence if for some $\sigma \in] 0, \rho]$ there exists a family $\left(q_{m, i}\right)_{\substack{1 \leq i \leq k(m) \\ m \in \mathbb{N}}}$ of nonnegative integers such that $\left(d\left(b_{m, i}, \sigma^{-}\right), q_{m, i}\right)_{\substack{1 \leq i \leq k(m) \\ m \in \mathbb{N}}}$ is a $T$-sequence.

Remark. An element of a polar sequence is either an element of $\bar{D} \backslash D$ or an element of a hole of $D$. 
Example. Suppose $K=\mathbb{C}_{p}$. Let $\Gamma=\left\{x \in K ; x^{p^{n}}=1\right.$, for some $n \geq 0\}$ and let $D=K \backslash \Gamma$. It is well known that elements of $\Gamma$ form a sequence $\left(b_{m, i}\right)_{\substack{1 \leq i \leq p^{m-1}(p-1) \\ m \in \mathbb{N}}}$ where $b_{m, i}$ lies in the circle of center 1 and diameter $p^{-\frac{1}{p^{m}(p-1)}}$. Then by [5, Proposition II.4], $\left(b_{m, i}\right)_{\substack{1 \leq i \leq p^{m-1}(p-1) \\ m \in \mathbb{N}}}$ is a $T$-polar sequence.

Notice that for all $r<p^{-\frac{1}{p(p-1)}}$ the set $D_{r}=\{x \in K ;|x-\gamma| \geq r \forall \gamma \in$ $\Gamma\}$ is not analytic $([\mathbf{5}])$.

\section{Definition of an analoid set.}

We build up our theory of strictly analytic functions on the so-called analoid sets.

Definition. $D$ will be said to be an analoid if $D$ satisfies:

1) Every $T$-polar sequence admits an empty $D$-beach.

2) Every monotonous distances holes sequences with a not empty $D$ beach has a superior limit-piercing strictly inferior to its diameter.

3) $D$ is open.

Remark. An analoid set is analytic. Indeed, suppose that an analoid set $D$ admits a $T$-sequence $\mathcal{S}=\left(T_{m, i}, q_{m, i}\right)_{\substack{1 \leq i \leq s(m) \\ m \in \mathbb{N}}}$ with a not empty $D$-beach. Let $R$ be the diameter of $\mathcal{S}$. For every $(m, i)(m \in \mathbb{N}$, $i \in\{1, \ldots, s(m)\})$, let $T_{m, i}=d\left(a_{m, i}, \rho_{m, i}^{-}\right)$. Since $D$ is analoid, $\mathcal{S}$ has a superior limit-piercing $\rho<R$. Hence, without loss of generality we assume that diameter of holes of $\mathcal{S}$ are upper bounded by $\sigma \in] \rho, R[$. Let $\left(d\left(b_{m, j}, \sigma^{-}\right)\right)_{\substack{1 \leq j \leq l(m) \\ m \in \mathbb{N}}}$ be the sequence of disks of diameter $\sigma$ such that every hole $T_{m, i}(m \in \mathbb{N}, 1 \leq i \leq s(m))$ is included in some $d\left(b_{m, j}, \sigma^{-}\right)$and that every disk $d\left(b_{m, j}, \sigma^{-}\right)(m \in \mathbb{N}, 1 \leq j \leq l(m))$ contains some $T_{m, i}$. For every $(m, j)(m \in \mathbb{N}, 1 \leq j \leq l(m))$ we denote by $\mathcal{I}_{m, j}$ the set of $(m, i)$ such that $T_{m, i} \subset d\left(b_{m, j}, \sigma^{-}\right)$and we put $p_{m, j}=\sum_{(m, i) \in \mathcal{I}_{m, j}} q_{m, i}$. Then, by [6, Proposition 35.4], the weighted sequence $\mathcal{S}^{\prime}=\left(d\left(b_{m, j}, \sigma^{-}\right), p_{m, j}\right)_{\substack{1 \leq j \leq l(m) \\ m \in \mathbb{N}}}$ is a $T$-sequence and therefore $D$ admits a $T$-polar sequence with a not empty $D$-beach, a contradiction with the hypothesis " $D$ is analoid". 


\section{Examples.}

Definitions. Let $D$ be a set that contains at least two points.

$D$ is called quasi-connected $([\mathbf{1 1}])$ if for any two points $x, y \in D$, the set $\{|z-x| ; z \in K \backslash D,|z-x| \leq|y-x|\}$ is finite.

A quasi-connected set $D$ is called regular ([10]) (resp. completely regu$\operatorname{lar}[\mathbf{1 3}])$ if for any two points $x, y \in D$ and any $r \in\left|K^{*}\right|$ with $r \leq|y-x|$, the set $(K \backslash D) \cap d(a, r)$ can be covered by countably (resp. finitely) many open balls with radius $r$.

We recall that the notion of regular quasi-connected set was first given by Krasner $([\mathbf{1 1}])$ when the residue class field of $K$ is not countable.

Quasi-connected sets (and particulary regular and completely regular quasi-connected sets) are analoids. Indeed, on one hand, it is well known that quasi-connected sets are open. On the other hand, since for all $x, y$ in a quasi-connected set $D$, the set $\{|z-x| ; z \in K \backslash D,|z-x| \leq|y-x|\}$ is finite, we see that $D$ has neither monotonous distances holes sequences nor polar sequence with a not empty $D$-beach and consequently it is an analoid.

But generally, analoid sets are not quasi-connected. Indeed, an analoid may have monotonous distances holes sequence and polars sequence with a not empty beach, which is not true for quasi-connected sets.

Let $\left(a_{n}\right)_{n \in \mathbb{N}}$ be an increasing distances sequence in the disk $d(0,1)$ of limit 1 satisfying $\sum_{n=0}^{\infty}-\log \left|a_{n}\right|<+\infty$ and let $\left(\rho_{n}\right)_{n \in \mathbb{N}}$ be a sequence in ]0,1[ such that $\limsup _{n \rightarrow \infty} \rho_{n}<1$. Let $D=d(0,2) \backslash\left(\bigcup_{n \in \mathbb{N}} d\left(a_{n}, \rho_{n}^{-}\right)\right)$. Clearly the holes of $D$ are of the form $d\left(a_{n}, \rho_{n}^{-}\right)$. According to [6, Proposition 36.5], such a set is analytic and then we see that $D$ is an analoid.

Remarks. i) One can't have an increasing covering of such a set $D$ by admissible sets either in the sense of [Fresnel Van der Put and Morita] or in the sense of [Karlowski and Ullrich]. Consequently, one can't define analytic functions on $D$ in these different senses.

ii) We notice that the class of analoid sets is not stable by intersection, but we define a subclass of "special" analoid sets which is so and which makes covering of every analoid set. 


\section{D-admissible sets.}

A hole $T=d\left(a, r^{-}\right)$of $D$ will be said to be circled if $d(a, r) \cap D=\emptyset$.

$D$ will be said to be peripherally circled if $D \cap C(a, \operatorname{diam}(D))=\emptyset$, for some $a \in D$, (i.e. $D$ is included in a class of $d(a, \operatorname{diam}(D))$.

Let $D$ be an analoid and let $U \subset D$. Then $U$ will be said to be $D$-admissible if $U$ is empty or if it satisfies the following conditions:

a) $U$ is closed and bounded.

b) $U$ is analytic and correctly pierced.

c) $U$ is well pierced.

d) Every circled hole of $D$ included in $\widetilde{U}$ is strictly included in a hole of $U$.

e) If $D$ is peripherally circled then $\widetilde{U} \varsubsetneqq \widetilde{D}$.

Remarks. i) Since $D$-admissible sets are well pierced, they are open too.

ii) A $D$-admissible set is an analoid.

iii) In general an analoid set $D$ is not $D$-admissible.

Definition. A $G$-topology on a set $X$ consist of:

a) A system $\mathcal{G}$ of subsets in $X$, called admissible open subsets.

b) A family $\{\operatorname{Cov}(U), U \in \mathcal{G}\}$ of systems of coverings called admissible coverings, where $\operatorname{Cov}(U)$, for each $U \in \mathcal{G}$, consists of coverings $\left(U_{i}\right)_{i \in I}$ of $U$ by sets $U_{i} \in \mathcal{G}, U_{i} \subset U$, satisfying the following conditions:

i) If $U, V \in \mathcal{G}$ then $U \cap V \in \mathcal{G}$.

ii) If $U \in \mathcal{G}$ then $\{U\} \in \operatorname{Cov}(U)$.

iii) If $U \in \mathcal{G},\left\{U_{i}\right\}_{i \in I} \in \operatorname{Cov}(U)$ and $\left\{V_{i j}\right\}_{j \in J} \in \operatorname{Cov}\left(U_{i}\right) \forall i \in I$, then $\left\{V_{i j}\right\}_{i \in I, j \in J} \in \operatorname{Cov}(U)$.

iv) If $U, V \in \mathcal{G}, V \subset U$ and $\left\{U_{i}\right\}_{i \in I} \in \operatorname{Cov}(U)$ then $\left\{V \cap U_{i}\right\}_{i \in I} \in$ $\operatorname{Cov}(V)$

Theorem 1. Let $D$ be an analoid. Let $\mathcal{G}$ be the system of $D$-admissible sets and for each $U \in \mathcal{G}$, let $\operatorname{Cov}(U)$ be the system of finite coverings of $U$ by subset of $U$ which are elements of $\mathcal{G}$.

Then $(\mathcal{G},\{\operatorname{cov}(U) ; U \in \mathcal{G}\})$ forms a $G$-topology on $D$.

The proof of this theorem first requires Lemmas 2, 3, 4, 5 and Theorems 6,7 . 
Lemma 2. Let $\mathcal{S}=\left(T_{m, i}, q_{m, i}\right)_{\substack{1 \leq i \leq k(m) \\ m \in \mathbb{N}}}$ be a weighted sequence of diameter $r$ and of monotony $\left(d_{m}\right)_{m \in \mathbb{N}}$. For each $m \in \mathbb{N}$, we put $q_{m}=\sum_{i=1}^{k(m)} q_{m, i}$. If $\mathcal{S}$ is a T-sequence, then $\sum_{m=0}^{+\infty} q_{m}\left|\log r-\log d_{m}\right|=+\infty$. Moreover, if $\sum_{m=0}^{+\infty} q_{m}\left|\log r-\log d_{m}\right|=+\infty$ and if $\mathcal{S}$ has a bounded perturbations sequence, then $\mathcal{S}$ is a $T$-sequence.

Proof: First we suppose $\left(d_{m}\right)_{m \in \mathbb{N}}$ increasing. If $\mathcal{S}$ is a $T$-sequence, then by definition we have $\lim _{m \rightarrow \infty}\left(\sum_{j=0}^{m-1} q_{j}\left(\log d_{m}-\log d_{j}\right)\right)=+\infty$ and therefore we have

$$
\lim _{m \rightarrow \infty}\left(\sum_{j=0}^{m} q_{j}\left(\log r-\log d_{j}\right)\right)=+\infty .
$$

We now suppose $\sum_{m=0}^{+\infty} q_{m}\left(\log r-\log d_{m}\right)=+\infty$ and that $\mathcal{S}$ has a perturbations sequence bounded by $\lambda$. By [6, Lemma 36.2], we have $\lim _{m \rightarrow \infty}\left(\sum_{j=0}^{m-1} q_{j}\left(\log d_{m}-\log d_{j}\right)\right)=+\infty . \quad$ For each $m \in \mathbb{N}$ and $i \in\{1, \ldots, k(m)\}$ we put $T_{m, i}=d\left(a_{m, i}, \rho_{m, i}^{-}\right)$. The sequence $\left(\omega_{m}\right)_{m \in \mathbb{N}}$ defined as

$$
\begin{aligned}
& \omega_{m}=\left(\sum_{j=0}^{m-1} q_{j}\left(\log d_{m}-\log d_{j}\right)\right) \\
&-\max _{1 \leq i \leq s(m)}\left(q_{m, j} \log \left(\frac{d_{m}}{\rho_{m, j}}\right)+\sum_{\substack{j \neq i \\
1 \leq j \leq k(m)}} q_{m, j}\left(\log d_{m}-\log \left|a_{m, j}-a_{m, i}\right|\right)\right),
\end{aligned}
$$

satisfies $\omega_{m} \geq\left[\left(\sum_{j=0}^{m-1} q_{j}\left(\log d_{m}-\log d_{j}\right)\right)-\lambda\right] . \quad$ So, we have $\lim _{m \rightarrow+\infty} w_{m}=+\infty$ and then $\mathcal{S}$ is a $T$-sequence. This finishes proving the lemma when $\left(d_{m}\right)_{m \in \mathbb{N}}$ is increasing. We show this symmetrically when $\left(d_{m}\right)_{m \in \mathbb{N}}$ is decreasing. 
Lemma 3. Let $\mathcal{S}=\left(T_{m, i}, q_{m, i}\right)_{\substack{1 \leq i \leq k(m) \\ m \in \mathbb{N}}}$ be a correctly pierced $T$ sequence of diameter $r$ and of inferior limit-piercing $\rho$. There exists an idempotent $T$-sequence of the form $\left(T_{m, i}, \mu_{m, i}\right)_{\substack{1 \leq i \leq k(m) \\ m \in \mathbb{N}}}$ with a bounded perturbations sequence.

Proof: Let $\rho$ (resp. $\sigma$ ) be the inferior (resp. superior) limit-piercing of $\mathcal{S}$. For each $(m, i)(1 \leq i \leq k(m), m \in \mathbb{N})$ we put $\rho_{m, i}=\operatorname{diam}\left(T_{m, i}\right)$. Since $\mathcal{S}$ is correctly pierced, there exists $N \in \mathbb{N}$ such that $\rho \leq \rho_{m, i} \leq \sigma$ whenever $i=1, \ldots, k(m)$ and $m \geq N$. Then the proof of [6, Theorem 43.2] shows that there exists an idempotent $T$-sequence of the form $\left(T_{m, i}, \mu_{m, i}\right)_{\substack{1 \leq i \leq k(m) \\ m \geq N}}$ whose perturbations sequence $\left(\omega_{m}\right)_{m \in \mathbb{N}}$ satisfies

$$
\omega_{m} \leq 4 \log \left(\frac{r}{\rho}\right), \forall m \geq N .
$$

For all $(m, i)(1 \leq i \leq k(m), m<N)$ we put $\mu_{m, i}=0$, and then $\left(T_{m, i}, \mu_{m, i}\right)_{\substack{1 \leq i \leq k(m) \\ m \in \mathbb{N}}}$ is an idempotent $T$-sequence with bounded perturbations sequence. This ends the proof of Lemma 3.

Lemma 4. Let $\mathcal{S}=\left(T_{n, i}, 1\right)_{\substack{1 \leq i \leq k(n) \\ n \in \mathbb{N}}}$ be an idempotent $T$-sequence with a bounded perturbations sequence. Let $\mathcal{S}^{\prime}=\left(T_{m, i}^{\prime}\right)_{\substack{1 \leq i \leq k^{\prime}(m) \\ m \in \mathbb{N}}}$ and $\mathcal{S}^{\prime \prime}=\left(T_{m, i}^{\prime \prime}\right)_{1 \leq i \leq k^{\prime \prime}(m)}$ be two cofiltring sequences such that $\left(T_{n, i}\right)_{\substack{1 \leq i \leq k(n) \\ n \in \mathbb{N}}}=\mathcal{S}^{\prime} \cup \mathcal{S}^{\prime \prime}$. Then there exists sequences $\left(\mu_{m, i}\right)_{\substack{1 \leq i \leq k^{\prime}(m) \\ m \in \mathbb{N}}}$ and $\left(\nu_{m, i}\right)_{\substack{1 \leq i \leq k^{\prime \prime}(m) \\ m \in \mathbb{N}}}$ of elements in $\{0,1\}$ such that at least one of the two weighted sequences $\left(T_{m, i}^{\prime}, \mu_{m, i}\right)_{\substack{1 \leq i \leq k^{\prime}(m) \\ m \in \mathbb{N}}}$ or $\left(T_{m, i}^{\prime \prime}, \nu_{m, i}\right)_{\substack{1 \leq i \leq k^{\prime \prime}(m) \\ m \in \mathbb{N}}}$ is an idempotent $T$-sequence.

Proof: Without loss of generality, we may assume $\mathcal{S}$ increasing. By Lemma 2 we have

$$
\sum_{m=0}^{+\infty} k(m)\left(\log r-\log d_{m}\right)=+\infty
$$


For each $(m, i)(m \in \mathbb{N}$ and $1 \leq i \leq k(m))$ (resp. $(m, i)(m \in \mathbb{N}$ and $\left.1 \leq i \leq k^{\prime}(m)\right)$, resp. $(m, i)\left(m \in \mathbb{N}\right.$ and $\left.\left.1 \leq i \leq k^{\prime \prime}(m)\right)\right)$, we put $T_{m, i}=$ $d\left(a_{m, i}, \rho_{m, i}{ }^{-}\right)\left(\operatorname{resp} . T_{m, i}^{\prime}=d\left(a_{m, i}^{\prime}, \rho_{m, i}^{\prime}{ }^{-}\right)\right.$, resp. $\left.T_{m, i}^{\prime \prime}=d\left(a_{m, i}^{\prime \prime}, \rho_{m, i}^{\prime \prime}{ }^{-}\right)\right)$. As $\mathcal{S}$ has a bounded perturbations sequence there exists $\lambda \in \mathbb{R}$ such that

(2) $\sup _{m \in \mathbb{N}}\left[\max _{1 \leq i \leq k(m)}\left(\log \left(\frac{d_{m}}{\rho_{m, i}}\right)\right.\right.$

$$
\left.+\sum_{\substack{j \neq i \\ 1 \leq j \leq k(m)}}\left(\log d_{m}-\log \left|a_{m, j}-a_{m, i}\right|\right)\right] \leq \lambda .
$$

We define $\left(\mu_{m, i}\right)_{\substack{1 \leq i \leq k^{\prime}(m) \\ m \in \mathbb{N}}}$ and $\left(\nu_{m, i}\right)_{\substack{1 \leq i \leq k^{\prime \prime}(m) \\ m \in \mathbb{N}}}$ as follows:

$\forall m \in \mathbb{N}$ and $1 \leq i \leq k^{\prime}(m)$, if $T_{m, i}^{\prime} \in \mathcal{S}$ then $\mu_{m, i}=1$ else $\mu_{m, i}=0$.

$\forall m \in \mathbb{N}$ and $1 \leq i \leq k^{\prime \prime}(m)$, if $T_{m, i}^{\prime \prime} \in \mathcal{S} \nu_{m, i}=1$, else $\nu_{m, i}=0$.

Let $q^{\prime}(m)=\sum_{i=1}^{k^{\prime}(m)} \mu_{m, i}$ and $q^{\prime \prime}(m)=\sum_{i=1}^{k^{\prime \prime}(m)} \nu_{m, i}$. Obviously, for all $m \in \mathbb{N}$ we have, $k(m) \leq q^{\prime}(m)+q^{\prime \prime}(m)$, then (1) implies that at least one of the two series $\sum_{m=0}^{+\infty} q^{\prime}(m)\left(\log r-\log d_{m}\right), \sum_{m=0}^{+\infty} q^{\prime \prime}(m)\left(\log r-\log d_{m}\right)$ diverges.

We suppose $\sum_{m=0}^{+\infty} q^{\prime}(m)\left(\log r-\log d_{m}\right)=+\infty$. We obviously have the relation

$$
\begin{gathered}
\sum_{\substack{j \neq i \\
1 \leq j \leq k^{\prime}(m)}} \mu_{m, j}\left(\log d_{m}-\log \left|a_{m, j}^{\prime}-a_{m, i}^{\prime}\right|\right) \\
\leq \sum_{\substack{j \neq i \\
1 \leq j \leq k(m)}}\left(\log d_{m}-\log \left|a_{m, j}-a_{m, i}\right|\right)
\end{gathered}
$$

for each $(m, i)\left(m \in \mathbb{N}, i \in\left\{1 \leq i \leq k^{\prime}(m)\right\}\right)$. 
Hence we have

$$
\begin{array}{r}
\sup _{m \in \mathbb{N}}\left[\operatorname { m a x } _ { 1 \leq i \leq k ^ { \prime } ( m ) } \left(\mu_{m, i} \log \left(\frac{d_{m}}{\rho_{m, i}^{\prime}}\right)\right.\right. \\
\left.\left.+\sum_{\substack{j \neq i \\
1 \leq j \leq k^{\prime}(m)}} \mu_{m, j}\left(\log d_{m}-\log \left|a_{m, j}^{\prime}-a_{m, i}^{\prime}\right|\right)\right]\right] \\
\sup _{m \in \mathbb{N}}\left[\max _{1 \leq i \leq k(m)}\left(\log _{\substack{\operatorname{mag}_{m}\left(\frac{d_{m}}{\rho_{m, i}}\right) \\
+}} \log d_{m}-\log \left|a_{m, j}-a_{m, i}\right|\right)\right] \leq \lambda .
\end{array}
$$

So the weighted sequence $\left(T_{m, i}^{\prime}, \mu_{m, i}\right)_{\substack{1 \leq i \leq k^{\prime}(m) \\ m \in \mathbb{N}}}$ has a bounded perturbations sequence and then by Lemma 2 , it is an idempotent $T$ sequence.

Lemma 5. Let $D^{\prime}$ and $D^{\prime \prime}$ be correctly pierced closed analytic sets such that $D^{\prime} \cap D^{\prime \prime} \neq \emptyset$. Then a hole of $D^{\prime} \cap D^{\prime \prime}$ is either a hole of $D^{\prime}$ or a hole of $D^{\prime \prime}$.

Proof: Let $T=d\left(a, r^{-}\right)$be a hole of $D^{\prime} \cap D^{\prime \prime}$. Since $a$ belongs to a hole of $D^{\prime}$ or $D^{\prime \prime}$, without loss of generality, we may assume that $a$ belongs to a hole $T^{\prime}$ of $D^{\prime}$. Moreover, if $a$ also belongs to a hole $T^{\prime \prime}$ of $D^{\prime \prime}$, we may assume that $\operatorname{diam}\left(T^{\prime}\right) \geq \operatorname{diam}\left(T^{\prime \prime}\right)$. We will check that $T=T^{\prime}$. It is clear that $T^{\prime} \subset T$; then if we suppose $\operatorname{diam}\left(T^{\prime}\right)<\operatorname{diam}(T)$, we see that the disk $d\left(a, \operatorname{diam}(T)^{-}\right)$admits a partition $\mathcal{P}$ by a family of holes of $D^{\prime}$ and $D^{\prime \prime}$. [12, Propositions 1.2 and 2.5] show that there exists $\left.t \in\right] 0, \operatorname{diam}(T)[$, $b \in d\left(a, r^{-}\right)$and an increasing idempotent $T$-sequence $\mathcal{S}=\left(T_{n}, 1\right)_{n \in \mathbb{N}}$ with holes in $\mathcal{P}$, of center $b$ and diameter $t$. Hence as $D^{\prime}$ and $D^{\prime \prime}$ are correctly pierced and as a hole of $\mathcal{S}$ is a hole of $D^{\prime}$ or a hole of $D^{\prime \prime}$, we can also assume $\mathcal{S}$ to be correctly pierced. Therefore by Lemma 3 we can assume $\mathcal{S}$ to have a bounded perturbations sequence. Using 
Lemma 4, from this $T$-sequence, either we may extract an idempotent $T$-sequence of holes of $D^{\prime}$ or we may extract an idempotent $T$-sequence of holes of $D^{\prime \prime}$. Since this $T$-sequence is increasing and its diameter satisfies $t<\operatorname{diam}(T)$, it has a not empty beach. Then this contradicts the hypothesis " $D^{\prime}$ and $D^{\prime \prime}$ are analytic sets".

Theorem 6. Let $D^{\prime}$ and $D^{\prime \prime}$ be correctly pierced analytic sets such that $D^{\prime} \cap D^{\prime \prime} \neq \emptyset$. Then $D^{\prime} \cap D^{\prime \prime}$ is a correctly pierced analytic set.

Proof: By Lemma 5 it is obvious that $D^{\prime} \cap D^{\prime \prime}$ is correctly pierced. By Theorem 0, we have to show that $D^{\prime} \cap D^{\prime \prime}$ doesn't have $T$-sequences with a not empty $D^{\prime} \cap D^{\prime \prime}$-beach. Let us suppose that $D^{\prime} \cap D^{\prime \prime}$ admits a $T$-sequence $\mathcal{S}$ with a not empty $D^{\prime} \cap D^{\prime \prime}$-beach. Since $D^{\prime} \cap D^{\prime \prime}$ is correctly pierced, Lemma 3 shows that we can associate to $\mathcal{S}$ an idempotent $T$ sequence with a bounded perturbations sequence. By Lemmas 4 and 5 , either $D^{\prime}$ or $D^{\prime \prime}$ admits an idempotent $T$-sequence with a not empty beach. But this contradicts the hypothesis " $D^{\prime}$ and $D^{\prime \prime}$ are analytic sets".

Theorem 7. Let $D$ be an analoid and let $U$ and $V$ be D-admissible sets. Then $U \cap V$ is D-admissible.

Proof: By definition of $D$-admissible sets, if $U \cap V$ is empty then it is $D$-admissible. Now, suppose that $U \cap V$ is not empty. Then by Theorem 6 , it is analytic and correctly pierced. Further, it is clear that $U \cap V$ is closed and bounded. Besides we know by Lemma 5 that a hole of $U \cap V$ is either a hole of $U$ or a hole of $V$. Thus, since $U$ and $V$ are well pierced, so is $U \cap V$.

Let $T$ be a circled hole of $D$ which is included in $\widetilde{U \cap V}$. As $\widetilde{U \cap V} \subset \widetilde{U}$ and as $\widetilde{U \cap V} \subset \widetilde{V}$, we see that $T \subset \widetilde{U}$ and $T \subset \widetilde{V}$. Then, since $U$ and $V$ are $D$-admissibles, $T$ is strictly included in a hole of $U$ and a hole of $V$. Thus by Lemma 5, we see that $T$ is strictly included in a hole of $U \cap V$. Finally, if $D$ is peripherally circled, then both $\widetilde{U}$ and $\widetilde{V}$ are strictly included in $\widetilde{D}$. Hence, $\widetilde{U \cap V}$ is strictly included in $\widetilde{D}$.

Proof of Theorem 1: Condition i) in the definition of a $G$-topology is satisfied by Theorem 7 . The other conditions are clearly satisfied by Theorem 7 and by our choice of $\mathcal{G}$ and of $\operatorname{cov}(U)$ for $U \in \mathcal{G}$.

Let $H$ be the presheaf on $D$ which, to each $D$-admissible $U$, associates $H(U)$. 
Theorem 8. The presheaf $H$ is a sheaf for the G-topology on D.

Proof: This is a consequence of the following lemma.

Lemma 9. Let $U_{1}$ and $U_{2}$ be correctly pierced closed analytic sets such that $U_{1} \cap U_{2} \neq \emptyset$. Let $f_{1} \in H\left(U_{1}\right), f_{2} \in H\left(U_{2}\right)$ such that

$$
f_{1} / U_{1} \cap U_{2}=f_{2} / U_{1} \cap U_{2} .
$$

Then, there exists $f \in H\left(U_{1} \cup U_{2}\right)$ such that $f / U_{j}=f_{j}(j=1,2)$.

Proof: By Lemma 5 a hole of $U_{1} \cap U_{2}$ is either a hole of $U_{1}$ or a hole of $U_{2}$. Hence Lemma 9 holds by [14, Theorem 8.3].

Remark. In a next paper we will study properties of this sheaf.

Let's now prove that an analoid admits a covering by an increasing sequence of $D$-admissible sets.

Lemma 10. Let $\mathcal{S}=\left(d\left(a_{m, i}, \rho_{m, i}^{-}\right), q_{m, i}\right)_{\substack{1 \leq i \leq s(m) \\ m \in \mathbb{N}}}$, and $\mathcal{S}^{\prime}=\left(d\left(b_{m, i}, \rho_{m, i}^{\prime-}\right), q_{m, i}\right)_{\substack{1 \leq i \leq s(m) \\ m \in \mathbb{N}}}$ be two weighted idempotent sequences of diameter $R$ and let $\delta>0$ and $r<R$ such that $\forall m, n \in \mathbb{N}, \forall i \in$ $\{1, \ldots, s(m)\}, \forall j \in\{1, \ldots, s(n)\} \quad i \neq j$, we have $\delta \leq \rho_{m, i} \leq r, \delta \leq$ $\rho_{m, i}^{\prime} \leq r$ and $\left|a_{m, i}-a_{n, j}\right|=\left|b_{m, i}-b_{n, j}\right|$.

Then $\mathcal{S}$ is a $T$-sequence if and only if $\mathcal{S}^{\prime}$ is a $T$-sequence.

Proof: Let $m \in \mathbb{N}$ and let $j \in\{1, \ldots, s(m)\}$. We put

$$
A_{m, j}=\prod_{\substack{i \neq j \\ 1 \leq i \leq s(m)}}\left(\frac{d_{m}}{\left|a_{m, i}-a_{m, j}\right|}\right)^{q_{m, i}}=\prod_{\substack{i \neq j \\ 1 \leq i \leq s(m)}}\left(\frac{d_{m}}{\left|b_{m, i}-b_{m, j}\right|}\right)^{q_{m, i}}
$$

and if $\left(d_{n}\right)_{n \in \mathbb{N}}$ is increasing (resp. decreasing) we put

$$
B_{m}=\prod_{n=1}^{m-1}\left(\frac{d_{n}}{d_{m}}\right)^{q_{n}}
$$

(resp.

$$
\left.B_{m}=\prod_{n=1}^{m-1}\left(\frac{d_{m}}{d_{n}}\right)^{q_{n}}\right)
$$


By definition, $\mathcal{S}$ (resp. $\mathcal{S}^{\prime}$ ) is a $T$-sequence if and only if

$$
\lim _{m \rightarrow \infty}\left(\sup _{1 \leq j \leq s(m)}\left[\left(\frac{d_{m}}{\rho_{m, j}}\right)^{q_{m, j}} A_{m, j}\right] B_{m}\right)=0
$$

(resp.

$$
\lim _{m \rightarrow \infty}\left(\sup _{1 \leq j \leq s(m)}\left[\left(\frac{d_{m}}{\rho_{m, j}^{\prime}}\right)^{q_{m, j}} A_{m, j}\right] B_{m}\right)=0
$$

According to the hypothesis of the lemma, obviously there exist $\alpha, \beta \in \mathbb{R}_{+}$and $N \in \mathbb{N}$ such that

$$
\begin{aligned}
& \alpha^{q_{m, i}} \leq\left(\frac{d_{m}}{\rho_{m, i}}\right)^{q_{m, i}} \leq \beta^{q_{m, i}}, \quad \text { and } \\
& \alpha^{q_{m, i}} \leq\left(\frac{d_{m}}{\rho_{m, i}^{\prime}}\right)^{q_{m, i}} \leq \beta^{q_{m, i}}, \quad \forall i \in\{1, \ldots, s(m)\} .
\end{aligned}
$$

We now suppose that $\mathcal{S}$ is a $T$-sequence; consequently $\mathcal{S}$ satisfies (1). But since each $q_{m, i}$ lies in $\{0,1\}(m \in \mathbb{N}, 1 \leq i \leq s(m))$, by (1) and (3), for every sequence $\left(x_{m}\right)_{m \in \mathbb{N}}$ such that $x_{m} \in\{1, \ldots, s(m)\}$, we have

$$
\lim _{m \rightarrow \infty} A_{m, x_{m}} B_{m}=0 .
$$

In particular, if $\left(x_{m}\right)_{m \in \mathbb{N}}$ is such that

$$
\left(\frac{d_{m}}{\rho_{m, x_{m}}^{\prime}}\right)^{q_{m, x_{m}}} A_{m, x_{m}}=\sup _{1 \leq j \leq s(m)}\left[\left(\frac{d_{m}}{\rho_{m, j}^{\prime}}\right)^{q_{m, j}} A_{m, j}\right]
$$

we see that using (4) and (5) we have

$$
\lim _{m \rightarrow \infty}\left(\sup _{1 \leq j \leq s(m)}\left[\left(\frac{d_{m}}{\rho_{m, j}^{\prime}}\right)^{q_{m, j}} A_{m, j}\right] B_{m}\right)=0 .
$$

We have a symmetric proof when $\mathcal{S}^{\prime}$ is a $T$-sequence.

Definition. Two cofiltring weighted sequences $\mathcal{S}=\left(d\left(a_{m, i}, \rho_{m, i}^{-}\right), q_{m, i}\right)_{\substack{1 \leq i \leq s(m) \\ m \in \mathbb{N}}}$ and $\mathcal{S}^{\prime}=\left(d\left(a_{m, i}, \rho_{m, i}^{\prime-}\right), q_{m, i}\right)_{\substack{1 \leq i \leq s(m) \\ m \in \mathbb{N}}}$
will be said to be similar if they have the same inferior and superior limit-piercing. 
Corollary 11. Let $\mathcal{S}$ and $\mathcal{S}^{\prime}$ be similar weighted sequences. Then $\mathcal{S}$ is correctly pierced if and only if $\mathcal{S}^{\prime}$ is correctly pierced.

Besides, if $\mathcal{S}$ is correctly pierced, then $\mathcal{S}$ is an idempotent $T$-sequence if and only if $\mathcal{S}^{\prime}$ is an idempotent $T$-sequence.

Lemma 12. Let $U_{1}, U_{2}$ be infraconnected sets such that

$$
\delta\left(U_{1}, U_{2}\right) \leq \min \left(\operatorname{diam}\left(U_{1}\right), \operatorname{diam}\left(U_{2}\right)\right)
$$

Then $U_{1} \cup U_{2}$ is infraconnected.

Proof: Let $a, b \in U_{1} \cup U_{2}$ and let $r_{1}, r_{2} \in \mathbb{R}$ such that $0<r_{1}<r_{2}<$ $|a-b|$. We just have to check that $\left(U_{1} \cup U_{2}\right) \cap \Gamma\left(a, r_{1}, r_{2}\right) \neq \emptyset$.

If $a, b \in U_{1}$ or $a, b \in U_{2}$, then since both $U_{1}$ and $U_{2}$ are infraconnected, we have $U_{1} \cap \Gamma\left(a, r_{1}, r_{2}\right) \neq \emptyset$ or $U_{2} \cap \Gamma\left(a, r_{1}, r_{2}\right) \neq \emptyset$ and therefore $\left(U_{1} \cup U_{2}\right) \cap \Gamma\left(a, r_{1}, r_{2}\right) \neq \emptyset$.

Now we assume that $a \in U_{1}$ and $b \in U_{2}$. First suppose, $r_{1}<\operatorname{diam}\left(U_{1}\right)$. Then there exists $a^{\prime} \in U_{1}$ such that $r_{1}<\left|a-a^{\prime}\right| \leq \operatorname{diam}\left(U_{1}\right)$. But since $U_{1}$ is infraconnected and $a, a^{\prime} \in U_{1}$, we have $U_{1} \cap \Gamma\left(a, r_{1}, \min \left(r_{2}, \mid a-\right.\right.$ $\left.\left.a^{\prime} \mid\right)\right) \neq \emptyset$. Consequently $\left(U_{1} \cup U_{2}\right) \cap \Gamma\left(a, r_{1}, r_{2}\right) \neq \emptyset$. Now suppose $r_{1} \geq \operatorname{diam}\left(U_{1}\right)$. We first assume $U_{2} \cap \widetilde{U_{1}} \neq \emptyset$; then for every $b^{\prime} \in U_{2} \cap \widetilde{U_{1}}$, we have $\Gamma\left(a, r_{1}, r_{2}\right)=\Gamma\left(b^{\prime}, r_{1}, r_{2}\right)$. So, since $U_{2}$ is infraconnected and $0<r_{1}<r_{2}<\left|b-b^{\prime}\right|$, we have $U_{2} \cap \Gamma\left(b^{\prime}, r_{1}, r_{2}\right) \neq \emptyset$ and therefore $\left(U_{1} \cup U_{2}\right) \cap \Gamma\left(a, r_{1}, r_{2}\right) \neq \emptyset$.

Finally, we assume $U_{2} \cap \widetilde{U_{1}}=\emptyset$. Then it is clear that $\delta\left(U_{1}, U_{2}\right)=$ $\inf _{x \in U_{2}}(|x-a|)$. But as $r_{1} \geq \operatorname{diam}\left(U_{1}\right)$ and $\delta\left(U_{1}, U_{2}\right) \leq \min \left(\operatorname{diam}\left(U_{1}\right)\right.$, $\left.\operatorname{diam}\left(U_{2}\right)\right)$, there exists $b^{\prime} \in U_{2}$ such that $\left|b^{\prime}-a\right|<r_{2}$. Since $U_{2}$ is infraconnected, we have $\Gamma\left(b^{\prime},\left|b^{\prime}-a\right|, r_{2}\right) \cap U_{2} \neq \emptyset$; but as $\Gamma\left(a,\left|b^{\prime}-a\right|, r_{2}\right)=$ $\Gamma\left(b^{\prime},\left|b^{\prime}-a\right|, r_{2}\right) \subset \Gamma\left(a, r_{1}, r_{2}\right)$, therefore $\left(U_{1} \cup U_{2}\right) \cap \Gamma\left(a, r_{1}, r_{2}\right) \neq \emptyset$.

We have a symmetric proof when $a \in U_{2}$ and $b \in U_{1}$. This ends the proof of Lemma 12 .

Lemma 13. Let $U_{1}$ and $U_{2}$ be infraconnected sets such that $U_{1} \cup U_{2}$ is infraconnected. Then $\widetilde{U_{1}} \subset \widetilde{U_{2}}$ or $\widetilde{U_{2}} \subset \widetilde{U_{1}}$.

Proof: Suppose that $\widetilde{U_{1}} \not \subset \widetilde{U_{2}}$ and $\widetilde{U_{2}} \not \subset \widetilde{U_{1}}$. By ultrametricity it is seen that $\delta\left(\widetilde{U_{1}}, \widetilde{U_{2}}\right)>\max \left(\operatorname{diam}\left(\widetilde{U_{1}}\right), \operatorname{diam}\left(\widetilde{U_{2}}\right)\right)$ and $\delta\left(\widetilde{U_{1}}, \widetilde{U_{2}}\right)=|a-b|$ for all $a \in U_{1}$ and $b \in U_{2}$. Let $r_{1}, r_{2} \in \mathbb{R}_{+}$be such that $\operatorname{diam}\left(\widetilde{U_{1}}\right)<$ $r_{1}<r_{2}<|a-b|$; then we see that $\Gamma\left(a, r_{1}, r_{2}\right) \cap\left(\widetilde{U_{1}} \cup \widetilde{U_{2}}\right)=\emptyset$. But this contradicts the hypothesis that $U_{1} \cup U_{2}$ is infraconnected. 
Lemma 14. Let $U_{1}, U_{2}$ be infraconnected sets such that $U_{1} \cup U_{2}$ is infraconnected. Then a hole of $U_{1} \cup U_{2}$ is either a hole of $U_{1}$ or a hole of $U_{2}$.

Besides, if a hole of $U_{1} \cup U_{2}$ is included in $\widetilde{U_{1}}$ and $\widetilde{U_{2}}$ then it is included in a hole of $U_{1}$ and in a hole of $U_{2}$.

Proof: Let $T=d\left(a, r^{-}\right)$be a hole of $U_{1} \cup U_{2}$. By Lemma 13, we can assume without loss of generality that $\widetilde{U_{1}} \subset \widetilde{U_{2}}$. First we suppose that $T \subset \widetilde{U_{1}}$. Then it is obvious that $T$ is simultaneously included in a hole of $U_{1}$ and in a hole of $U_{2}$.

Since $r=\inf _{x \in U_{1} \cup U_{2}}(|x-a|)=\min \left(\inf _{x \in U_{1}}(|x-a|), \inf _{x \in U_{2}}(|x-a|)\right)$, it is seen that $T$ is either a hole of $U_{1}$ or a hole of $U_{2}$.

Now if we suppose that $T \not \subset \widetilde{U_{1}}$, then it is clear that $\delta\left(a, \widetilde{U_{1}}\right)>r$. But since $\delta\left(a, \widetilde{U_{1}}\right)=\delta\left(a, U_{1}\right)=\inf _{x \in U_{1}}(|x-a|)$, then obviously we have $r=\inf _{x \in U_{2}}(|x-a|)$ and as $T \subset \widetilde{U_{2}}, T$ is a hole of $U_{2}$.

Lemma 15. Let $D$ be an analoid and let $U_{1}, U_{2}$ be D-admissible sets without increasing T-sequence. Further, we assume that $U_{1} \cup U_{2}$ is correctly pierced.

a) If $U_{1} \cap U_{2} \neq \emptyset$, then $U_{1} \cup U_{2}$ is $D$-admissible.

b) If $\delta\left(U_{1}, U_{2}\right)=\operatorname{diam}\left(U_{1}\right)=\operatorname{diam}\left(U_{2}\right)$, then $U_{1} \cup U_{2}$ is D-admissible.

Proof: By Lemma 12, if $U_{1} \cap U_{2} \neq \emptyset$ or $\delta\left(U_{1}, U_{2}\right)=\operatorname{diam}\left(U_{1}\right)=$ $\operatorname{diam}\left(U_{2}\right)$ then $U_{1} \cup U_{2}$ is infraconnected. Besides, since $U_{1} \cup U_{2}$ is bounded, closed and correctly pierced, we only have to show that it is well pierced, analytic, that every circled hole of $D$ included in $\widetilde{U_{1} \cup U_{2}}$ is strictly included in a hole of $U_{1} \cup U_{2}$ and that if $D$ is peripherally circled, then $\widetilde{U_{1} \cup U_{2}} \varsubsetneqq \widetilde{D}$. The two last statements are obvious, because on one hand a circled hole of $D$ included in $\widetilde{U_{1} \cup U_{2}}$ is included in a hole of $U_{1} \cup U_{2}$. But by Lemma 14, a hole of $U_{1} \cup U_{2}$ is a hole of $U_{1}$ or a hole of $U_{2}$. Then, since both $U_{1}$ and $U_{2}$ are $D$-admissible sets, we have a strict inclusion. On the other hand, if $D$ is peripherally circled, then both $\widetilde{U_{1}}$ and $\widetilde{U_{2}}$ are strictly included in $\widetilde{D}$. Hence, $\widetilde{U_{1} \cup U_{2}}$ is strictly included in $\widetilde{D}$.

Since $U_{1}$ and $U_{2}$ are well pierced, then by Lemma 14 so is $U_{1} \cup U_{2}$.

We will show that $U_{1} \cup U_{2}$ is analytic. Indeed let us suppose that this is not true. So, by Theorem $0, U_{1} \cup U_{2}$ admits a $T$-sequence $\mathcal{S}$ with a not empty $U_{1} \cup U_{2}$-beach. But since $U_{1} \cup U_{2}$ is correctly pierced, by 
Lemma 3, we may assume that $\mathcal{S}$ is an idempotent $T$-sequence with a bounded perturbations sequence.

Lemmas 14 and 4 show that there exists a $T$-sequence $\mathcal{S}_{1}$ or $\mathcal{S}_{2}$ whose holes are holes of $U_{1}$ or holes of $U_{2}$ respectively. For example let us suppose $\mathcal{S}_{1}$ to be this $T$-sequence. Obviously $\mathcal{S}_{1}$ is decreasing because $U_{1}$ does not admit increasing $T$-sequences. Since $U_{1}$ is analytic, by Theorem 0 we see that $\mathcal{S}_{1}$ has an empty $U_{1}$-beach. Hence the $U_{1} \cup U_{2}$-beach of $\mathcal{S}$ is included in $U_{2}$. Let $a$ be an element of the $U_{1} \cup U_{2}$-beach of $\mathcal{S}$, then $a \in U_{2}$.

a) We assume $U_{1} \cap U_{2} \neq \emptyset$ and take $b \in U_{1} \cap U_{2}$. In particular we have $|a-b|>\operatorname{diam}(\mathcal{S})$. By Lemma 14, we see that from certain rank, the holes of $\mathcal{S}_{1}$ are included in holes of $U_{2}$. To such a hole of $U_{2}$ we associate the sum of the weights of holes of $\mathcal{S}_{1}$ that it contains. Hence, we obtain a weighted sequence of holes of $U_{2}$ which is, by [6, Proposition 35.4], a $T$-sequence. Thus $U_{2}$ admits a $T$-sequence with a not empty $U_{2}$-beach, which contradicts the hypothesis that $U_{2}$ is analytic.

b) Now we assume that

$$
\delta\left(U_{1}, U_{2}\right)=\operatorname{diam}\left(U_{1}\right)=\operatorname{diam}\left(U_{2}\right) .
$$

Since $\mathcal{S}_{1}$ is decreasing, it is clear that

$$
\operatorname{diam}\left(\mathcal{S}_{1}\right)<\operatorname{diam}\left(U_{1}\right)
$$

As $a \in U_{2}$, by $(2)$, we have $\delta\left(U_{1}, U_{2}\right) \leq \operatorname{diam}\left(\mathcal{S}_{1}\right)$ which is a contradiction with (1).

Thus $U_{1} \cup U_{2}$ is analytic and therefore it is $D$-admissible.

Notation. Henceforth, $K$ is supposed topologically separable. It is well known that such a field is not spherically complete $([\mathbf{1 4}])$ and we see that $\mathbb{C}_{p}$ satisfies such a conditions.

Definition. We will call prepierced filter on $D$ every monotonous filter on $D$ with center, less thin than a polar sequence.

Remark. Let $D$ be an infraconnected set of $K$ and let $\lambda<\operatorname{diam}(D)$. Then, since $K$ is separable, the family of disks $d\left(a, \lambda^{-}\right)$included in $\widetilde{D}$ which contain elements of $\bar{D} \backslash D$ is countable. Moreover, the family of disks included in $\widetilde{D}$ whose centers are centers of prepierced filters of diameter $\lambda$ is countable too. 
Lemma 16. Let $a \in K$ and $r>0$. Then a partition of $d\left(a, r^{-}\right)$by non-circumferenced disks is a singleton or infinite.

Proof: Suppose that the partition is not reduced to a singleton. Let $d\left(a, \rho^{-}\right)$be the element of the partition containing $a$. Then, we see that $\rho<r$. Since $K$ is algebraically closed, its valuation group is dense in $\mathbb{R}$. Then, let $b, c \in K$ such that $\rho<|b-a|<|c-a|<r$. Let $d\left(b, \rho_{b}^{-}\right)$and $d\left(c, \rho_{c}^{-}\right)$are the elements of the partition containing $b$ and $c$ respectively. Obviously we have $d\left(b, \rho_{b}^{-}\right) \cap d\left(c, \rho_{c}^{-}\right)=\emptyset$. Hence, the partition of $d\left(a, r^{-}\right)$is infinite.

Lemma 17. Let $D$ be an analoid. Let $d\left(a, r^{-}\right)$be such that $d(a, r) \varsubsetneqq \widetilde{D}$. Let $\lambda \in] 0, r\left[\right.$ and $\lambda \notin|K|$. Let $\left(d\left(a_{n}, \rho_{n}{ }^{-}\right)\right)_{n \in \mathbb{N}}$ be the family of holes of $D$ included in $d\left(a, r^{-}\right)$, of diameter superior or equal to $\lambda$. Let $\left(d\left(b_{n}, \mu_{n}{ }^{-}\right)\right)_{n \in \mathbb{N}}$ be the family of holes of $D$ included in $d\left(a, r^{-}\right)$, of diameter strictly inferior to $\lambda$. Let $\left(c_{n}\right)_{n \in \mathbb{N}}$ be a sequence of elements of $D$ such that, for all $n \in \mathbb{N}, c_{n}$ is center of a prepierced filter of $D$ of diameter $\lambda$. Let $\left(d\left(d_{n}, \lambda^{-}\right)\right)_{n \in \mathbb{N}}$ be the family of disks which contain an element of $\bar{D} \backslash D$.

If $\left(s_{n}\right)_{n \in \mathbb{N}}$ and $\left(t_{n}\right)_{n \in \mathbb{N}}$ are sequences in $\mathbb{R}_{+}$of limits zero, such that

$$
\begin{aligned}
& d\left(a, r^{-}\right)=\bigcup_{n \in \mathbb{N}}\left(d\left(a_{n}, \rho_{n}\left(1+s_{n}\right)^{-}\right)\right. \\
&\left.\cup d\left(b_{n}, \lambda\left(1+t_{n}\right)^{-}\right) \cup d\left(c_{n}, \lambda^{-}\right) \cup d\left(d_{n}, \lambda^{-}\right)\right),
\end{aligned}
$$

then there exists $m \in \mathbb{N}$ such that

$$
\begin{aligned}
& d\left(a, r^{-}\right)=d\left(a_{m}, \rho_{m}\left(1+s_{m}\right)^{-}\right) \text {or } \\
& d\left(a, r^{-}\right)=d\left(b_{m}, \lambda\left(1+t_{m}\right)^{-}\right) .
\end{aligned}
$$

Proof: Let $\mathcal{T}_{1}=\left\{d\left(a_{n}, \rho_{n}\left(1+s_{n}\right)^{-}\right) ; n \in \mathbb{N}\right\}$, let $\mathcal{T}_{2}=\left\{d\left(b_{n}, \lambda(1+\right.\right.$ $\left.\left.\left.t_{n}\right)^{-}\right) ; n \in \mathbb{N}\right\}$, let $\mathcal{T}_{3}=\left\{d\left(c_{n}, \lambda^{-}\right) ; n \in \mathbb{N}\right\}$ and let $\mathcal{T}_{4}=\left\{d\left(d_{n}, \lambda^{-}\right) ; n \in\right.$ $\mathbb{N}\}$. We put $\mathcal{T}=\mathcal{T}_{1} \cup \mathcal{T}_{2} \cup \mathcal{T}_{3} \cup \mathcal{T}_{4}$. We will denote by $\mathcal{R}$ the relation defined on $\mathcal{T}$ by $U \mathcal{R} V$ if there exists $W \in \mathcal{T}$ such that $U \subset W$ and $V \subset W$. This relation is obviously seen to be an equivalence relation on $\mathcal{T}$. For every $U \in \mathcal{T}$, we put $\widehat{U}=\bigcup_{V \in \bar{U}} V$ where $\bar{U}$ is the equivalence class of $U$.

We will show that for each $U \in \mathcal{T}$, there exists $V \in \bar{U}$ such that $V=\widehat{U}$ and that there is only one equivalence class with respect to the relation $\mathcal{R}$. 
Suppose that for certain $U \in \mathcal{T}$ we have $V \varsubsetneqq \widehat{U}$ for all $V \in \bar{U}$. Therefore there exists a sequence $\left(V_{\alpha(n)}\right)_{n \in \mathbb{N}}$ in $\frac{\bar{U}}{U}$, strictly increasing with respect to the inclusion, whose limit diameters is equal to $\operatorname{diam}(\widehat{U})$. Without loss of generality we may assume that the sequence $\left(V_{\alpha(n)}\right)_{n \in \mathbb{N}}$ either is in $\mathcal{T}_{1}$ or is in $\mathcal{T}_{2}$ or is in $\mathcal{T}_{3}$ or is in $\mathcal{T}_{4}$.

First, we assume that $V_{\alpha(n)} \in \mathcal{T}_{1}, \forall n \in \mathbb{N}$ and we write

$$
V_{\alpha(n)}=d\left(a_{\alpha(n)}, \rho_{\alpha(n)}\left(1+s_{\alpha(n)}\right)^{-}\right), \quad \forall n \in \mathbb{N}
$$

Then, since $\lim _{n \rightarrow+\infty} s_{n}=0$, we see that

$$
\lim _{n \rightarrow \infty} \rho_{\alpha(n)}=\operatorname{diam}(\widehat{U})
$$

By $\left[6\right.$, Theorem 3.1], we may extract from $\left(a_{\alpha(n)}\right)_{n \in \mathbb{N}}$ a sequence $\left(a_{\beta(n)}\right)_{n \in \mathbb{N}}$ which either is convergent or is an equal distances sequence or is a monotonous distances sequence.

Since $\rho_{n} \geq \lambda$ for all $n \in \mathbb{N}$ and $d\left(a_{n}, \rho_{n}^{-}\right) \cap d\left(a_{m}, \rho_{m}\right)=\emptyset$, for all $n \neq m$, clearly the sequence $\left(a_{\beta(n)}\right)_{n \in \mathbb{N}}$ can't be convergent.

If $\left(a_{\beta(n)}\right)_{n \in \mathbb{N}}$ is an equal distances sequence of value $A$, then since $\left(V_{\alpha(n)}\right)_{n \in \mathbb{N}}$ is a strictly increasing sequence, we obviously have

$$
A<\operatorname{diam}(\widehat{U})
$$

Moreover, as the holes of $D$ are disjointed, we have

$$
\rho_{\beta(n)} \leq A, \quad \forall n \in \mathbb{N}
$$

Then we see that (2) and (3) contradict (1).

If $\left(a_{\beta(n)}\right)_{n \in \mathbb{N}}$ is a monotonous distances sequence, then by (1) necessarily the sequence $\left(d\left(a_{\beta(n)}, \rho_{\beta(n)}^{-}\right)_{n \in \mathbb{N}}\right.$ is an increasing distances holes sequences, of diameter $\operatorname{diam}(\widehat{U})$ and of superior limit-piercing $\operatorname{diam}(\widehat{U})$. Then, since the sequence $\left(d\left(a_{\beta(n)}, \rho_{\beta(n)}^{-}\right)_{n \in \mathbb{N}}\right.$ has a not empty $D$-beach (because $\left.V_{\beta(n)} \subset \widehat{U} \subset d\left(a, r^{-}\right) \varsubsetneqq \widetilde{D}, \forall n \in \mathbb{N}\right)$, we see that this contradicts the hypothesis " $D$ is an analoid".

Second, suppose that $V_{\alpha(n)} \in \mathcal{T}_{2}, \forall n \in \mathbb{N}$. On one hand, since $\left(V_{\alpha(n)}\right)_{n \in \mathbb{N}}$ is strictly increasing, we see that $\operatorname{diam}(\hat{U})>\lambda$. On the other hand, since $\lim _{n \rightarrow+\infty} t_{n}=0$, we have $\operatorname{diam}(\hat{U})=\lambda$, which is impossible. 
Finally, $\left(V_{\alpha(n)}\right)_{n \in \mathbb{N}}$ can't be a strictly increasing sequence for the inclusion in $\mathcal{T}_{3}$ (resp. $\mathcal{T}_{4}$ ) because their elements are of diameter $\lambda$. Thus this finishes proving that for each $U \in \mathcal{T}$, there exists $V \in \bar{U}$ such that $V=\widehat{U}$. Hence we see that $d\left(a, r^{-}\right)$admits a partition by a family of elements of $\mathcal{T}$.

Let us suppose that this partition is not reduced to a singleton. Then, by Lemma 16 this partition is infinite. Let $t \in] \lambda, r[$. By $[\mathbf{1 2}$, Proposition 2.5] we deduce the existence of an idempotent increasing $T$-sequence $\mathcal{S}=\left(d\left(u_{m}, r_{m}^{-}\right), 1\right)_{\substack{1 \leq i \leq k(m) \\ m \in \mathbb{N}}}$ of diameter $t$ and whose elements belong to $\mathcal{T}$.

We will show that $\mathcal{S}$ is correctly pierced. Indeed, we notice that every element of $\mathcal{T}$ has a diameter superior to $\lambda$, and therefore the inferior limit-piercing of $\mathcal{S}$ is not zero. Moreover, by definition, every hole of $\mathcal{T}_{3}$ and $\mathcal{T}_{4}$ is of diameter $\lambda$. We also remark that, since $\lim _{n \rightarrow+\infty} t_{n}=0$, then every monotonous distances sequence in $\mathcal{T}_{2}$ has a superior limit-piercing equal to $\lambda$. On other hand, since $\lim _{n \rightarrow+\infty} s_{n}=0$ and $D \cap\left(K \backslash d\left(a, r^{-}\right)\right) \neq \emptyset$ every increasing distances sequence of holes in $\mathcal{T}_{1}$ has a not empty $D$ beach, and therefore it is correctly pierced (because $D$ is). Consequently, as $t>\lambda$ and as $\mathcal{T}=\mathcal{T}_{1} \cup \mathcal{T}_{2} \cup \mathcal{T}_{3} \cup \mathcal{T}_{4}, \mathcal{S}$ is correctly pierced too.

Let $b$ be a center of $\mathcal{S}$ and let $m \in \mathbb{N}$.

- If $d\left(u_{m}, r_{m}^{-}\right) \in \mathcal{T}_{1}$, then there exists $\gamma(m) \in \mathbb{N}$ such that $d\left(u_{m}, r_{m}^{-}\right)=d\left(a_{\gamma(m)}, \rho_{\gamma(m)}\left(1+s_{\gamma(m)}\right)^{-}\right)$and therefore we put $d\left(v_{m}, r_{m}^{\prime-}\right)=d\left(a_{\gamma(m)}, \rho_{\gamma(m)}^{-}\right)$.

- If $d\left(u_{m}, r_{m}^{-}\right) \in \mathcal{T}_{2}$, then there exists $\gamma(m) \in \mathbb{N}$ such that $d\left(u_{m}, r_{m}^{-}\right)=d\left(b_{\gamma(m)}, \lambda\left(1+t_{\gamma(m)}\right)^{-}\right)$and therefore we put $d\left(v_{m}, r_{m}^{\prime-}\right)=d\left(b_{\gamma(m)}, \lambda^{-}\right)$.

- If $d\left(u_{m}, r_{m}^{-}\right) \in \mathcal{T}_{3}$, then there exists $\gamma(m) \in \mathbb{N}$ such that $d\left(u_{m}, r_{m}^{-}\right)=d\left(c_{\gamma(m)}, \lambda^{-}\right)$. In this case, by hypothesis, $d\left(c_{\gamma(m)}, \lambda^{-}\right)$ is the disk of centers of a prepierced filter of diameter $\lambda$. Then we see that there exists $v_{m} \in \widetilde{D} \backslash D$ such that

$$
\left|u_{m}-v_{m}\right|<\left|u_{m}-b\right|
$$

So, if $v_{m}$ belongs to a hole of $D$ of diameter $\rho$, we put $d\left(v_{m}, r_{m}^{\prime}{ }^{-}\right)=$ $d\left(v_{m}, \rho^{-}\right)$; else (i.e. $\left.v_{m} \in \bar{D} \backslash D\right)$, we put $d\left(v_{m}, r_{m}^{\prime}{ }^{-}\right)=d\left(v_{m}, \lambda^{-}\right)$.

- If $d\left(u_{m}, r_{m}^{-}\right) \in \mathcal{T}_{4}$ then there does exist $\gamma(m) \in \mathbb{N}$ such that $d\left(u_{m}, r_{m}^{-}\right)=d\left(d_{\gamma(m)}, \lambda^{-}\right)$and then we put $d\left(v_{m}, r_{m}^{\prime-}\right)=d\left(d_{\gamma(m)}, \lambda^{-}\right)$. 
Hence we have obtained a weighted sequence $\mathcal{S}^{\prime}=\left(d\left(v_{m}, r_{m}^{\prime-}\right), 1\right)_{\substack{1 \leq i \leq k(m) \\ m \in \mathbb{N}}}$ which is cofiltring to $\mathcal{S}$ and satisfying

$$
\left|u_{m}-u_{n}\right|=\left|v_{m}-v_{n}\right|, \quad \forall m, n \in \mathbb{N} .
$$

We first see that $\mathcal{S}^{\prime}$ is increasing and has a not empty $D$-beach because $d\left(a, r^{-}\right) \varsubsetneqq \widetilde{D}$. But since $D$ is correctly pierced and $0<\lambda<t, \mathcal{S}^{\prime}$ is also correctly pierced.

Now we see that both $\mathcal{S}$ and $\mathcal{S}^{\prime}$ are cofiltring, correctly pierced, satisfy (5) and that $\mathcal{S}$ is an idempotent $T$-sequence. Then by Lemma $10, \mathcal{S}^{\prime}$ is an idempotent $T$-sequence. Therefore, by Lemma 3, we can assume that $\mathcal{S}^{\prime}$ has a bounded perturbations sequence. As each hole of $\mathcal{S}^{\prime}$ either is a hole of $D$ or contains an element of $\bar{D} \backslash D$, then Lemma 4 shows that $D$ admits either a $T$-sequence with a not empty $D$-beach or a $T$ polar sequence with a not empty $D$-beach. But this contradicts the hypothesis that $D$ has no $T$-polar sequences with a not empty $D$-beach. So, the partition of $d\left(a, r^{-}\right)$is reduced to a singleton, and therefore, there exists $T \in \mathcal{T}$ such that $T=d\left(a, r^{-}\right)$. But since $r>\lambda$, we see that there exists $m \in \mathbb{N}$ such that either $d\left(a, r^{-}\right)=d\left(a_{m}, \rho_{m}\left(1+s_{m}\right)^{-}\right)$or $d\left(a, r^{-}\right)=d\left(b_{m}, \lambda\left(1+t_{n}\right)^{-}\right)$. This ends the proof of Lemma 17.

Lemma 18. Let $D$ be an analoid, let $a, b \in D$ and let $r>0$ such that $r \leq|a-b|$. Then there exists a $D$-admissible $U_{a, r}$ containing $a$, of diameter $r$, without increasing $T$-sequences and such that every increasing distances sequence of holes of $U_{a, r}$ is correctly pierced.

Proof: Let $\lambda \in] 0, \min (r, \delta(a, K \backslash D))$ [ be such that $\lambda \notin|K|$. Let $\left(\mathcal{F}_{n}\right)_{n \in \mathbb{N}}$ be the sequence of prepierced filters of diameter $\lambda$, secant with $d\left(a, r^{-}\right)$.

Let $\left(d\left(a_{n}, \rho_{n}{ }^{-}\right)\right)_{n \in \mathbb{N}}$ be the family of holes of $D$ included in $d\left(a, r^{-}\right)$, of diameter superior or equal to $\lambda$, let $\left(d\left(b_{n}, \mu_{n}{ }^{-}\right)\right)_{n \in \mathbb{N}}$ be the family of holes of $D$, included in $d\left(a, r^{-}\right)$, of diameter strictly inferior to $\lambda$, let $\left(c_{n}\right)_{n \in \mathbb{N}}$ be such that for all $n \in \mathbb{N}, c_{n}$ is center of $\mathcal{F}_{n}$ and let $\left(d\left(d_{n}, \lambda^{-}\right)\right)_{n \in \mathbb{N}}$ be the family of disks of diameter $\lambda$ which contain elements of $(\bar{D} \backslash D) \cap d\left(a, r^{-}\right)$.

For all $n \in \mathbb{N}$, we put $u_{n}=\left|a-a_{n}\right|$. If $d\left(a_{n}, \rho_{n}{ }^{-}\right)$is circled, we have $u_{n}>\rho_{n}$. So, we may choose $\left.\varepsilon_{n} \in\right] 0, \frac{1}{n+1}\left[\right.$ satisfying $\rho_{n}\left(1+\varepsilon_{n}\right)<u_{n}$. If $d\left(a_{n}, \rho_{n}{ }^{-}\right)$is not circled, we put $\varepsilon_{n}=0$. 
For all $n \in \mathbb{N}$, we put $T^{1}{ }_{n}=d\left(a_{n}, \rho_{n}\left(1+\varepsilon_{n}\right)^{-}\right)$. Let $\mathcal{T}_{1}=\left\{T^{1}{ }_{n} ; n \in\right.$ $\mathbb{N}\}$, let $\mathcal{T}_{2}=\left\{d\left(b_{n}, \lambda^{-}\right) ; n \in \mathbb{N}\right\}$, let $\mathcal{T}_{3}=\left\{d\left(c_{n}, \lambda^{-}\right) ; n \in \mathbb{N}\right\}$, let $\mathcal{T}_{4}=$ $\left\{d\left(d_{n}, \lambda^{-}\right) ; n \in \mathbb{N}\right\}$ and let $\mathcal{T}=\mathcal{T}_{1} \cup \mathcal{T}_{2} \cup \mathcal{T}_{3} \cup \mathcal{T}_{4}$. We define $U_{a, r}$ as follows:

$$
U_{a, r}=\left[d\left(a, r^{-}\right)\right] \backslash\left[\bigcup_{n \in \mathbb{N}}\left(T^{1}{ }_{n} \cup d\left(b_{n}, \lambda^{-}\right) \cup d\left(c_{n}, \lambda^{-}\right) \cup d\left(d_{n}, \lambda^{-}\right)\right)\right] .
$$

By construction we have $a \in U_{a, r}$ and $\operatorname{diam}\left(U_{a, r}\right) \leq r$. Let us suppose that $\operatorname{diam}\left(U_{a, r}\right)<r$. Then for $\left.r_{1}, r_{2} \in\right] \max \left(\lambda, \operatorname{diam}\left(U_{a, r}\right)\right), r[$ and for $u \in K$ such that $r_{1}<|u-a|<r_{2}<|a-b|$, the disk $d\left(u,|a-u|^{-}\right)$ is a union of holes of $\mathcal{T}$. By Lemma 17, there exists $T \in \mathcal{T}$ such that $d\left(u,|a-u|^{-}\right)=T$. More precisely, as $\lambda<|u-a|$, we have $T \in \mathcal{T}_{1}$. Thus, the annulus $\Gamma\left(a, r_{1}, r_{2}\right)$ admits a partition $\mathcal{P}$ by elements of $\mathcal{T}_{1}$. Let $t \in] r_{1}, r_{2}[$. By [12, Proposition 2.5] there exists an idempotent increasing $T$-sequence $\mathcal{S}$, of diameter $t$ whose elements lie in $\mathcal{T}_{1}$. By hypothesis we have $\lim _{n \rightarrow \infty} \varepsilon_{n}=0$, so this sequence is similar to a sequence $\mathcal{S}_{D}$ of holes of $D$. The $D$-beach of $\mathcal{S}_{D}$ contains $b$ because $|a-b|>r$. Hence, since $D$ is correctly pierced, so is $\mathcal{S}_{D}$. Consequently, as $\mathcal{S}$ is a $T$-sequence, using Corollary 11, we see that $\mathcal{S}_{D}$ is a $T$-sequence, which is absurd because $D$ doesn't admit $T$-sequences with a not empty $D$-beach. Thus $\operatorname{diam}\left(U_{a, r}\right)=r$.

We will check that a hole of $U_{a, r}$ is an element of $\mathcal{T}$. Indeed, let $T$ be a hole of $U_{a, r}$. It is clearly seen that $T$ is a union of holes of $\mathcal{T}$. Then by Lemma 17, there exists $T^{\prime} \in \mathcal{T}$ such that $T=T^{\prime}$. We deduce that the diameters of holes of $U_{a, r}$ are superior to $\lambda$ and consequently, $U_{a, r}$ is well pierced. Besides, $U_{a, r}$ is closed by construction.

Next, given a monotonous distances (resp. weighted) sequence $\mathcal{S}$ of holes of $U_{a, r}$, we may denote by $\mathcal{S}_{1}$ (resp. $\mathcal{S}_{2}$, resp. $\mathcal{S}_{3}$, resp. $\mathcal{S}_{4}$ ) the subsequence which consists of the holes of $\mathcal{S}$ lying in $\mathcal{T}_{1}$ (resp. $\mathcal{T}_{2}$, resp. $\mathcal{T}_{3}$, resp. $\left.\mathcal{T}_{4}\right)$.

We will show that $U_{a, r}$ is correctly pierced. Then, since $U_{a, r}$ is well pierced, we only have to prove that every monotonous distances sequence $\mathcal{S}$ of holes of $U_{a, r}$, of diameter $\rho$, either has an empty $U_{a, r}$-beach or has a superior limit-piercing strictly inferior to $\rho$. Without loss of generality we may suppose that $\mathcal{S}$ is of center 0 . If $\mathcal{S}_{1}$ is infinite, then it is similar to a sequence $\mathcal{S}_{D}$ of holes of $D$. As $U_{a, r} \subset D$ and as $D$ is an analoid, we see that if $\mathcal{S}$ has a not empty $U_{a, r}$-beach, then $\mathcal{S}_{D}$ and $\mathcal{S}_{1}$ are correctly pierced. 
If $\mathcal{S}_{2}$ (resp. $\mathcal{S}_{3}$, resp. $\mathcal{S}_{4}$ ) is infinite, its superior limit-piercing is equal to $\lambda$. Obviously, we have $\rho \geq \lambda$. Suppose that $\rho=\lambda$, then there exists $n \in \mathbb{N}$ such that $0 \in d\left(c_{n}, \lambda^{-}\right)$; but since $\lambda \notin|K|$ and by definition of $U_{a, r}$, we have $U_{a, r} \cap d\left(c_{n}, \lambda\right)=\emptyset$. Thus $\mathcal{S}_{2}$ (resp. $\mathcal{S}_{3}$, resp. $\mathcal{S}_{4}$ ) has an empty $U_{a, r}$-beach. So, if $\mathcal{S}$ has a not empty $U_{a, r}$-beach, it is correctly pierced and consequently $U_{a, r}$ is correctly pierced. Besides, we see that every increasing distances sequence of holes of $U_{a, r}$ is correctly pierced. Indeed, if $\mathcal{S}$ is an increasing distances sequence of holes of $U_{a, r}$ with an empty beach, necessarily its diameter is $r$. Since $r>\lambda$ we see that if the superior limit-piercing of $\mathcal{S}$ is equal to $r$, then $\mathcal{S}_{1}$ is infinite and has a superior limit-piercing equal to $r$, hence $\mathcal{S}_{1}$ is similar to an increasing sequence of holes of $D$. But since $|a-b|>r$, this sequence has a not empty beach which contradicts the hypothesis " $D$ is an analoid".

Now we will check that $U_{a, r}$ doesn't have increasing $T$-sequences and that it doesn't admit decreasing $T$-sequences with a not empty $U_{a, r^{-}}$ beach. Indeed, let us suppose that $U_{a, r}$ admits an increasing (resp. decreasing) $T$-sequence $\mathcal{S}$ (resp. with a not empty $U_{a, r}$-beach) of center 0 . We know that such a sequence $\mathcal{S}$ is correctly pierced. Therefore by Lemma 3 we may assume that $\mathcal{S}$ is idempotent and has a bounded perturbations sequence. Hence, by Lemma $4, \mathcal{S}_{1}$ or $\mathcal{S}_{2}$ or $\mathcal{S}_{3}$ or $\mathcal{S}_{4}$ is a $T$-sequence. If $\mathcal{S}_{1}$ or $\mathcal{S}_{2}$ is a $T$-sequence, then as each hole of $\mathcal{T}_{1}$ and $\mathcal{T}_{2}$ contains a hole of $D$ and as $|b| \geq \operatorname{diam}(\mathcal{S}$ ) (resp. as $\mathcal{S}$ has a not empty $U_{a, r}$-beach), Lemma 10 shows that $D$ admits a $T$-sequence with a not empty $D$-beach, which contradicts the hypothesis that $D$ is analytic. If $\mathcal{S}_{3}$ is a $T$-sequence, then since each element of $\mathcal{T}_{3}$ is the set of centers of a prepierced filter of diameter $\lambda$, we can construct a monotonous distances sequence $\mathcal{S}_{D}$ of holes of $D$ such that both $\mathcal{S}_{3}$ and $\mathcal{S}_{D}$ satisfy the hypothesis of Lemma 10. But this implies that $D$ admits a $T$-sequence with a not empty $D$-beach, which is absurd because $D$ is analytic. Finally, if $\mathcal{S}_{4}$ is a $T$-sequence, then $D$ admits a $T$-polar sequence with a not empty $D$-beach, which is also absurd. Thus $U_{a, r}$ doesn't have any increasing $T$-sequences and doesn't admit any decreasing $T$-sequences with a not empty $U_{a, r}$-beach.

Thus using Theorem 0 we see that as any $T$-sequence of $U_{a, r}$ has an empty $U_{a, r}$-beach, $U_{a, r}$ is analytic. This ends the proof of Lemma 18.

Theorem 19. Let $D$ be an analoid and let $a, b \in D$ with $a \neq b$. Then there exists a $D$-admissible $U_{a, b}$ containing a and $b$, without increasing $T$-sequences. Moreover $U_{a, b}$ is of diameter $|a-b|$ and any increasing distances sequences of holes of $U_{a, b}$ is correctly pierced.

Proof: Let $r=|a-b|$. By Lemma 18, there exists a $D$-admissible $U_{a, r}$ (resp. $U_{b, r}$ ) containing $a$ (resp. $b$ ), of diameter $r$, without increasing 
$T$-sequences, such that every increasing distances sequence of holes of $U_{a, r}$ (resp. $U_{b, r}$ ) is correctly pierced.

Obviously we see that $\delta\left(U_{a, r}, U_{b, r}\right)=\operatorname{diam}\left(U_{a, r}\right)=\operatorname{diam}\left(U_{b, r}\right)$, and then by Lemma 12, $U_{a, r} \cup U_{b, r}$ is infraconnected and every decreasing distances sequence of holes of $U_{a, r} \cup U_{b, r}$ with a not empty $U_{a, r} \cup U_{b, r^{-}}$ beach is either a decreasing distances sequence of holes of $U_{a, r}$ with a not empty $U_{a, r}$-beach or a decreasing distances sequence of holes of $U_{b, r}$ with a not empty $U_{b, r}$-beach. But since $U_{a, r}$ and $U_{b, r}$ are correctly pierced, then such a sequence is correctly pierced. Since any increasing distances sequence of holes of $U_{a, r}$ (resp. $U_{b, r}$ ) is correctly pierced, by Lemma 14 , it is also true for any increasing distances sequence of holes of $U_{a, r} \cup U_{b, r}$. Thus $U_{a, r} \cup U_{b, r}$ is correctly pierced.

Finally, Lemma 15 shows that the set $U_{a, b}=U_{a, r} \cup U_{b, r}$ is $D$-admissible.

Theorem 20. Let $D$ be an analoid. Then there exists an increasing sequence $\left(U_{n}\right)_{n \in \mathbb{N}}$ of $D$-admissibles such that $D=\bigcup_{n \in \mathbb{N}} U_{n}$.

Proof: Let $a \in D$ and let $\left(a_{n}\right)_{n \in \mathbb{N}}$ be a dense sequence in $D$. For all $i \in$ $\mathbb{N}$, by Theorem 19 there exists a $D$-admissible $U_{a, a_{i}}$ of diameter $\left|a-a_{i}\right|$, without increasing $T$-sequences, which contains $a$ and $a_{i}$.

For all $n \in \mathbb{N}$, we put

$$
U_{n}=\bigcup_{i=0}^{n}\left(U_{a, a_{i}} \bigcup d\left(a_{i}, r_{i}\right)\right) \text {, with } r_{i}=\frac{1}{2} \min \left(\left|a-a_{i}\right|, \delta\left(a_{i}, K \backslash D\right)\right) \text {. }
$$

By Lemma 12, it is obvious that $U_{n}$ is infraconnected. On one hand, for all $i \in\{0,1, \ldots, n\}$, both $U_{a, a_{i}}$ and $d\left(a_{i}, r\right)$ are well pierced and then by Lemma $14, U_{n}$ is well pierced. On the other hand, by Theorem 19, we see that each increasing distances sequence of holes of $U_{a, a_{i}}$ is correctly pierced, hence by Lemma 14 , so is any increasing distances sequences of holes of $U_{n}$. This way, to show that $U_{n}$ is correctly pierced, we only have to check that every decreasing distances sequence of holes of $U_{n}$ with a not empty $U_{n}$-beach, of diameter $r$, has a superior limit-piercing strictly inferior to $r$. Let $\mathcal{S}$ be such a sequence and let $b$ an element of its $U_{n}$-beach. Obviously there exists $i_{0} \in\{0,1, \ldots, n\}$ such that $b \in$ $U_{a, a_{i} 0} \cup d\left(a_{i_{0}}, r_{i_{0}}\right)$. Since $\mathcal{S}$ is a decreasing distances sequence, naturally we have $r<\operatorname{diam}\left(U_{n}\right)$.

Then, if $|a-b|>r$, by Lemma 14 the holes of $\mathcal{S}$ are included in holes of $U_{a, a_{i}}$. So, we obtain a sequence of holes of $U_{a, a_{i}}$ which is cofiltring to $\mathcal{S}$ and has a not empty $U_{a, a_{i_{0}}}$-beach. But since $U_{a, a_{i_{0}}}$ is correctly pierced, 
we see that, in particular, its superior limit-piercing is strictly inferior to $r$. It follows that it is the same for the superior limit-piercing of $\mathcal{S}$. Now, if $|a-b| \leq r$, by ultrametricity we see that $a$ belongs to the beach of $\mathcal{S}$. Obviously there exists $i_{1} \in\{0,1, \ldots, n\}$ such that $\left|a-a_{i_{1}}\right|=\operatorname{diam}\left(U_{n}\right)$. Therefore we have $\left|b-a_{i_{1}}\right|>r$ and then by Lemma 14, from certain rank each hole of $\mathcal{S}$ is included in a hole of $U_{a, a_{i_{1}}}$. So, these holes of $U_{a, a_{i_{1}}}$ form a decreasing sequence cofiltring to $\mathcal{S}$, whose $U_{a, a_{i_{1}}}$-beach contains $a$. Moreover, its superior limit-piercing is strictly inferior to $r$ because $U_{a, a_{i_{1}}}$ is correctly pierced. Hence, $\mathcal{S}$ has a superior limit-piercing strictly inferior to $r$. This shows that $U_{n}$ is correctly pierced and then by Lemma $15, U_{n}$ is $D$-admissible.

Finally let $b \in D$; we will show that $b \in \bigcup_{n \in \mathbb{N}} U_{n}$. If $a=b$ then obviously $a \in U_{a, a_{0}}$. Else we take $\lambda=\delta(b, K \backslash D)$. Since $D$ is open we have $\lambda>0$. Then there exists $n \in \mathbb{N}$ such that $\left|b-a_{n}\right|<\min \left(\frac{\lambda}{3},|a-b|\right)$, therefore $b \in d\left(a_{n}, r_{n}\right)$ and it follows that $b \in U_{n}$. Thus we have $D=\bigcup_{n \in \mathbb{N}} U_{n}$ and $U_{n} \subset U_{n+1}$ for all $n \in \mathbb{N}$.

Definition and notation. Let $D$ be an analoid and let $f \in K^{D}$. Then we will say that $f$ is a strictly analytic function on $D$ if for any $D$-admissible $U$ the restriction $f / U$ of $f$ to $U$ is an element of $H(U)$.

We will denote by $\mathcal{A}(D)$ the set of strictly analytic functions on $D$.

Remarks. Here we obtain a definition of analytic functions over an important class of open sets. Obviously we may define strictly analytic functions over quasi-connected sets that, according to Example 3 in section II, form a subclass of the class of analoids.

Moreover, as the ground field is not spherically complete, it is well known that there exist many analytic functions in the sense of KrasnerRobba that may not be expanded in Taylor series in some disks ([14, p. 146]). On the other hand, analytic functions in our sense are always expanded in Taylor series in disks (see Proposition 23).

As notice in the preliminaries, in Fresnel-van der Put's theory, one considers infraconnected sets $D$ with a covering made of affinoid sets which are not necessarily infraconnected, and therefore analytic functions obtained by this way contain non trivial characteristic functions of certain subsets $D^{\prime}$ of $D$.

In [13] Morita defined analytic functions over the class of completely regular quasi-connected sets using Tate's theory of rigid analytic spaces ([17]). On such sets, this definition is equivalent to the one of Fresnel 
and Van Der Put. Over a completely regular quasi-connected set in a topologically separable field $K$, it is easy to show that our definition, Morita's definition and Fresnel-Van-Der-Put's definition are equivalent.

By an other process Karlowski and Ullrich ([10]) defined analytic functions on regular quasi-connected sets in an algebraically closed complete ultrametric field. We know that a regular quasi-connected set is quasiconnected and that a quasi-connected set in a field with a countable residue class field is regular. We notice that if we suppose $D$ to be quasiconnected in a topologically separable field $K$, our definition of analytic functions is not equivalent to Karlowski-Ullrich's definition. Indeed, in the example in page 416 of [10] they construct an unbounded analytic function $f$ (in sense of Karlowski and Ullrich) on a quasi-connected set $D$. But such a function does not belong to $\mathcal{A}(D)$, because since $D$ is $D$ admissible, if $f$ lied in $\mathcal{A}(D)$ it should lie in the Banach algebra $H(D)$. But, analytic functions in the sense of Karlowski and Ullrich are only defined on regular quasi-connected sets, which gives a restricted theory.

Example. Let $\left(a_{n}\right)_{n \in \mathbb{N}}$ and $\left(\alpha_{n}\right)_{n \in \mathbb{N}}$ be two sequences in $K$ such that $\left|a_{n}\right|<\left|a_{n+1}\right|$ and $\left|\alpha_{n}\right|<\left|\alpha_{n+1}\right|, \forall n \in \mathbb{N}$ and such that $\lim _{n \rightarrow \infty}\left|a_{n}\right|=1$, $\lim _{n \rightarrow \infty}\left|\alpha_{n}\right|=2, \sum_{n=0}^{\infty}-\log \left|a_{n}\right|<+\infty$ and $\sum_{n=0}^{\infty}\left(\log 2-\log \left|a_{n}\right|\right)<+\infty$. Let $\left(\rho_{n}\right)_{n \in \mathbb{N}}$ be a sequence in $] 0,1\left[\right.$ such that $\lim _{n \rightarrow \infty} \rho_{n}=0$. Let $D=d(0,3) \backslash\left(\left(\bigcup_{n \in \mathbb{N}} d\left(a_{n}, \rho_{n}^{-}\right)\right) \bigcup\left\{\alpha_{n} ; n \in \mathbb{N}\right\}\right)$. Clearly, the holes of $D$ are of the form $d\left(a_{n}, \rho_{n}^{-}\right)$. By [6, Proposition 36.5], such a set does not admit $T$-polar sequences. Hence, we see that $D$ is an analoid.

Choose $\left(\lambda_{n}\right)_{n \in \mathbb{N}}$ and $\left(\mu_{n}\right)_{n \in \mathbb{N}}$ two sequences in $K$ such that $0<$ $\lim _{n \rightarrow \infty} \frac{\left|\lambda_{n}\right|}{\rho_{n}}<+\infty$ and $\lim _{n \rightarrow \infty}\left|\mu_{n}\right|=0$ and put $f=\sum_{n=0}^{\infty} \frac{\lambda_{n}}{x-a_{n}}+\sum_{n=0}^{\infty} \frac{\mu_{n}}{x-\alpha_{n}}$. Let $U$ be a $D$-admissible set. Since $U$ is well pierced, there exists $\sigma>0$ such that diameter of holes of $U$ are minorated by $\sigma$. As $\lim _{n \rightarrow \infty}\left|\lambda_{n}\right|=$ $\lim _{n \rightarrow \infty}\left|\mu_{n}\right|=0$, we see that both sequences $\sum_{n=0}^{N} \frac{\lambda_{n}}{x-a_{n}}$ and $\sum_{n=0}^{N} \frac{\mu_{n}}{x-\alpha_{n}}$ converge uniformly on $U$ (when $N$ tends to $+\infty$ ). So, for any $D$ admissible set $U, \sum_{n=0}^{\infty} \frac{\lambda_{n}}{x-a_{n}}$ and $\sum_{n=0}^{\infty} \frac{\mu_{n}}{x-\alpha_{n}}$ belong to $H(U)$ and consequently, they belong to $\mathcal{A}(D)$. As $f=\sum_{n=0}^{\infty} \frac{\lambda_{n}}{x-a_{n}}+\sum_{n=0}^{\infty} \frac{\mu_{n}}{x-\alpha_{n}}$, we 
see that $f$ belongs to $\mathcal{A}(D)$. Obviously, $\sum_{n=0}^{\infty} \frac{\lambda_{n}}{x-a_{n}}, \sum_{n=0}^{\infty} \frac{\mu_{n}}{x-\alpha_{n}}$ and $f$ don't belong to $H(D)$.

\section{Properties of strictly analytic functions.}

Proposition 21. Let $D$ be an analoid.

1) The set $\mathcal{A}(D)$ is a $K$-algebra. It contains $H(D)$.

Let $D^{\prime}$ be an analoid satisfying $D^{\prime} \subset D$.

2) Every $D^{\prime}$-admissible set is a D-admissible set.

3) The restriction of an element of $\mathcal{A}(D)$ to $D^{\prime}$ is an element of $\mathcal{A}\left(D^{\prime}\right)$.

Proof: The assertions 1) and 2) are obvious. Let $f \in \mathcal{A}(D)$ and $U$ be a $D^{\prime}$-admissible set. By 2) we see that the restriction of $f$ to $U$ is an element of $H(U)$. Hence $f$ is a strictly analytic function on $D^{\prime}$.

Proposition 22. Let $D$ be an analoid and $f \in \mathcal{A}(D)$. Then $f^{\prime} \in \mathcal{A}(D)$.

Proof: Let $U$ be $D$-admissible. Since $f / U$ belongs to $H(U)$ and since $U$ is well pierced, by [6, Corollary 19.2] one has $f^{\prime} / U \in H(U)$. Hence $f^{\prime}$ belongs to $\mathcal{A}(D)$.

Proposition 23. Let $D$ be an analoid, let $a \in D$ and let $r \in \mathbb{R}^{*}+$ such that $d(a, r) \subset D$ (resp. $\left.d\left(a, r^{-}\right) \subset D\right)$. Let $f \in \mathcal{A}(D)$. In $d(a, r)$ (resp. $\left.d\left(a, r^{-}\right)\right), f(x)$ can be expanded in the form $\sum_{n=0}^{\infty} a_{n}(x-a)^{n}$ with $\lim _{n \rightarrow \infty}\left|a_{n}\right| s^{n}=0, \forall s<r$.

Proof: We see that for all $s<r$ the disk $d(a, s)$ is $D$-admissible. So, by definition of strictly analytic functions on $D$, the restriction of $f$ to $d(a, s)$ is an element of $H(d(a, s))$. Therefore, by [6, Corollary 13.2], in $d(a, s)$ $f$ is equal to a power series of the form $\sum_{n=0}^{\infty} a_{n}(x-a)^{n}$. Obviously such a serie doesn't depend on $s$ and satisfies $\lim _{n \rightarrow \infty}\left|a_{n}\right| s^{n}=0$, for all $s<r$.

Proposition 24. Let $D$ be an analoid and let $\Gamma\left(a, r, r^{\prime}\right) \subset D$. The restriction of an element of $\mathcal{A}(D)$ to $\Gamma\left(a, r, r^{\prime}\right)$ is a Laurent series convergent in $\Gamma\left(a, r, r^{\prime}\right)$. 
Proof: Let $f \in \mathcal{A}(D)$ and let $s, s^{\prime}>0$ are such that $r<s<s^{\prime}<r^{\prime}$. Then $\Delta\left(a, s, s^{\prime}\right)$ is a $D$-admissible set. So the restriction of $f$ to $\Delta\left(a, s, s^{\prime}\right)$ is an element of $H\left(\Delta\left(a, s, s^{\prime}\right)\right)$. By the Mittag-Leffler Theorem [6], in $\Delta\left(a, s, s^{\prime}\right)$ we have $f(x)=\sum_{n=1}^{+\infty} \frac{a_{n}}{(x-a)^{n}}+\sum_{n=0}^{+\infty} b_{n}(x-a)^{n}$, where $\sum_{n=1}^{+\infty} \frac{a_{n}}{(x-a)^{n}}$ converges in $K \backslash d\left(a, s^{-}\right)$and $\sum_{n=0}^{+\infty} b_{n}(x-a)^{n}$ converges in $d\left(a, s^{\prime}\right)$. Clearly we see that $\sum_{n=1}^{+\infty} \frac{a_{n}}{(x-a)^{n}}$ and $\sum_{n=0}^{+\infty} b_{n}(x-a)^{n}$ don't depend on $s$ and $s^{\prime}$.

For all $x \in \Gamma\left(a, r, r^{\prime}\right), \sum_{n=1}^{+\infty} \frac{a_{n}}{(x-a)^{n}}$ and $\sum_{n=0}^{+\infty} b_{n}(x-a)^{n}$ converge and $f(x)=\sum_{n=1}^{+\infty} \frac{a_{n}}{(x-a)^{n}}+\sum_{n=0}^{+\infty} b_{n}(x-a)^{n}$.

Proposition 25. Let $a \in K$ and $f=\sum_{n=0}^{\infty} a_{n}(x-a)^{n}$ of radius of convergence superior or equal to $r$. Then $f \in \mathcal{A}\left(d\left(a, r^{-}\right)\right)$.

Proof: Since $d\left(a, r^{-}\right)$is peripherally circled, for every $d\left(a, r^{-}\right)$-admissible $U$ there exists $s \in] 0, r[$ such that $\widetilde{U} \subset d(a, s)$. Then, as $f$ belongs to $H(d(a, s))$, we see that $f$ belongs also to $H(U)$ and this proves that $f \in \mathcal{A}\left(d\left(a, r^{-}\right)\right)$.

Proposition 26. Let $f=\sum_{n=0}^{+\infty} \frac{a_{n}}{(x-a)^{n}}$ be a Laurent series convergent in $K \backslash d(a, r)$. Then $f \in \mathcal{A}(K \backslash d(a, r))$.

Proof: Let $D=K \backslash d(a, r)$. Obviously we see that for all $D$-admissible set $U$ there exists $s>r$ such that $U \subset K \backslash d\left(a, s^{-}\right)$. Since $f \in H(K \backslash$ $\left.d\left(a, s^{-}\right)\right)$we have $f \in H(U)$ and consequently $f \in \mathcal{A}(D)$.

Remark. $\mathcal{A}(K)$ is the algebra of power series of infinite radius of convergence. Therefore the Liouville Theorem [1] holds: a bounded strictly analytic function on $K$ is constant. 
Definition. A set $D \subset K$ is said to be circled if at least one of the following statements is satisfied:

i) $D$ admits a circled hole.

ii) $D$ is peripherally circled.

Otherwise it is said uncircled.

Proposition 27. Let $D$ be an analoid. The following statements are equivalent:

a) $\mathcal{A}(D)=H(D)$.

b) $D$ is $D$-admissible.

Proof: Clearly b) implies a).

If $D$ is not $D$-admissible then, since it is an analoid, either $D$ is not closed or is not bounded or is not well pierced or is circled.

First suppose that $D$ is not closed and, without loss of generality, that $0 \in \bar{D} \backslash D$ (resp. we suppose that $D$ is unbounded). Let $\left(a_{n}\right)_{n \in \mathbb{N}}$ be a sequence in $K^{*}$ such that $\lim _{n \rightarrow \infty} \sqrt[n]{\left|a_{n}\right|}=0$. Then, such a sequence satisfies $\lim _{n \rightarrow \infty} \frac{\left|a_{n}\right|}{s^{n}}=0$ (resp. $\lim _{n \rightarrow \infty}\left|a_{n}\right| s^{n}=0$ ) for all $s>0$. Hence we see that the series $f=\sum_{n=0}^{\infty} \frac{a_{n}}{x^{n}}$ (resp. $\left.f=\sum_{n=0}^{\infty} a_{n} x^{n}\right)$ belongs to $\mathcal{A}(K \backslash\{0\})$ (resp. $\mathcal{A}(K)$ ). In particular the restriction $f / D$ of $f$ to $D$ belongs to $\mathcal{A}(D)$, but it is well known that $f / D$ is not in $H(D)$.

If $D$ is not well pierced, by [6, Theorem 19.7] $H(D)$ is not stable by derivation. Hence, according to Proposition $22, H(D)$ is strictly included in $\mathcal{A}(D)$.

Finally, suppose that $D$ admits a circled hole that we suppose (without loss of generality) equal to $d\left(0, r^{-}\right)$(resp. we suppose that $D$ is peripherally circled of diameter $S)$. Then let $\left(a_{n}\right)_{n \in \mathbb{N}}$ be a sequence in $K^{*}$ satisfying

(1) $\left(\frac{\left|a_{n}\right|}{r^{n}}\right)_{n \in \mathbb{N}}\left(\operatorname{resp} .\left(\left|a_{n}\right| S^{n}\right)_{n \in \mathbb{N}}\right)$ is unbounded.

(2) $\lim _{n \rightarrow \infty} \sqrt[n]{\left|a_{n}\right|}=r\left(\right.$ resp. $\left.\lim _{n \rightarrow \infty} \sqrt[n]{\left|a_{n}\right|}=\frac{1}{S}\right)$. 
Then such a sequence satisfies $\lim _{n \rightarrow \infty} \frac{\left|a_{n}\right|}{s^{n}}=0 \forall s>r$ (resp. $\lim _{n \rightarrow \infty}\left|a_{n}\right| s^{n}=0$ $\forall s<S)$. Hence the serie $f=\sum_{n=0}^{\infty} \frac{a_{n}}{x^{n}}$ (resp. $f=\sum_{n=0}^{\infty} a_{n} x^{n}$ ) belongs to $\mathcal{A}(K \backslash d(0, r))$ (resp. $\left.\mathcal{A}\left(d\left(0, S^{-}\right)\right)\right)$and consequently its restriction to $D$ belongs to $\mathcal{A}(D)$. But according to (1), we see that $f / D$ is not in $H(D)$ and this ends the proof of Proposition 27.

\section{Analytic continuation.}

In Theorem 28 we will show that strictly analytic functions on an analoid of $K$ satisfy the property of analytic continuation.

Theorem 28. Let $D$ be an analoid in $K, f \in \mathcal{A}(D), a \in D$ and $r>0$. If $f(x)=0$ for all $x \in d(a, r) \cap D$ then $f(x)=0$ for all $x \in D$.

Proof: Indeed, let $b \in D$. We will show that $f(b)=0$. By Theorem 19, there exists a $D$-admissible $U_{a, b}$ which contains $a$ and $b$. It is obvious that $f(x)=0$ for all $x \in d(a, r) \cap U_{a, b}$. So, since $f / U_{a, b} \in H\left(U_{a, b}\right)$ and since $U_{a, b}$ is analytic, we see that $f(x)=0$ for all $x \in U_{a, b}$. In particular, we have $f(b)=0$.

7. The differential equation $y^{\prime}=f y$ in algebras $\mathcal{A}(D)$.

Let $D$ be an analoid and let $f \in \mathcal{A}(D)$. We denote by $\mathcal{E}(f)$ the differential equation $y^{\prime}=f y$ with $y \in \mathcal{A}(D)$ and by $\mathcal{S}(f)$ the $K$-vector space of solutions $h \in \mathcal{A}(D)$.

Theorem 29. $\mathcal{S}(f)$ has dimension 0 or 1 .

Proof: Assume that $\mathcal{S}(f)$ has a not identically zero solution $g$. Let $a \in D$ such that $g(a) \neq 0$. Let $h$ be another not identically zero solution. Let $b \in D$, then by Theorem 19 , there exists a $D$-admissible set $U_{a, b}$ containing $a$ and $b$. For every $l \in \mathcal{A}(D)$, let $l_{a, b}$ be the restriction of $l$ to $U_{a, b}$. Then $g_{a, b}, h_{a, b}$ are solutions of the equation $y^{\prime}=f_{a, b} y$ in $H\left(U_{a, b}\right)$. But since $g(a) \neq 0$, by [6, Theorem 55.4], we have $h(b)=\frac{h(a)}{g(a)} g(b)$. Therefore, if we put $\lambda=\frac{h(a)}{g(a)}$, we see that $h(x)=\lambda g(x)$ for all $x \in D$.

Example. Let $\left(a_{n}\right)_{n \in \mathbb{N}}$ and $\left(\alpha_{n}\right)_{n \in \mathbb{N}}$ be two sequences in $K$ satisfying $\left|a_{n}\right|<\left|a_{n+1}\right|,\left|\alpha_{n}\right|<\left|\alpha_{n+1}\right| \forall n \in \mathbb{N}, \lim _{n \rightarrow \infty}\left|a_{n}\right|=1, \lim _{n \rightarrow \infty}\left|\alpha_{n}\right|=2$, $\sum_{n=0}^{\infty}-\log \left|a_{n}\right|<+\infty$ and $\sum_{n=0}^{\infty}\left(\log 2-\log \left|\alpha_{n}\right|\right)<+\infty$. Let $\left(\rho_{n}\right)_{n \in \mathbb{N}}$ be a 
sequence in $] 0,1\left[\right.$ such that $\lim _{n \rightarrow \infty} \rho_{n}=0$ and choose $\left(\lambda_{n}\right)_{n \in \mathbb{N}}$ and $\left(\mu_{n}\right)_{n \in \mathbb{N}}$ two sequences in $K$ such that $0<\lim _{n \rightarrow \infty} \frac{\left|\lambda_{n}\right|}{\rho_{n}}, \lim _{n \rightarrow \infty} \mu_{n}=0$ and $\left|\lambda_{n}\right|<\rho_{n}$ $\forall n \in \mathbb{N}$.

For every $n \in \mathbb{N}$, we put $b_{n}=a_{n}+\lambda_{n}$ and $\beta_{n}=\alpha_{n}+\mu_{n}$.

Let $\left.D=d(0,3) \backslash\left(\bigcup_{n \in \mathbb{N}} d\left(a_{n}, \rho_{n}^{-}\right)\right) \bigcup\left\{\alpha_{n} ; n \in \mathbb{N}\right\} \bigcup\left\{\beta_{n} ; n \in \mathbb{N}\right\}\right)$. According to [6, Lemma 4 and Proposition 36.5], we check that $D$ doesn't admit $T$-polar sequences and therefore is analoid.

Let $f=\sum_{n=0}^{\infty} \frac{\lambda_{n}}{\left(x-a_{n}\right)\left(x-b_{n}\right)}+\sum_{n=0}^{\infty} \frac{\mu_{n}}{\left(x-\alpha_{n}\right)\left(x-\beta_{n}\right)}$. Since any $D$ admissible set $U$ is well pierced, and since $\lim _{n \rightarrow \infty} \lambda_{n}=\lim _{n \rightarrow \infty} \mu_{n}=0$, it is easy to show that the restriction of $f$ to $U$ is an element of $H(U)$ and consequently $f \in \mathcal{A}(D)$.

Let $\mathcal{E}(f)$ be the differential equation

$$
y^{\prime}=f y \text { in } \mathcal{A}(D) .
$$

The function $g=\prod_{n=0}^{\infty}\left(\frac{x-b_{n}}{x-a_{n}}\right) \prod_{n=0}^{\infty}\left(\frac{x-\alpha_{n}}{x-\beta_{n}}\right)$ lies in $\mathcal{A}(D)$ and is a solution of $\mathcal{E}(f)$. Indeed, first we show that given a $D$-admissible set $U$, the product $h_{m}=\prod_{n=0}^{m}\left(\frac{x-b_{n}}{x-a_{n}}\right)$ converges uniformly on $U$. As $U$ is well pierced we may choose $\sigma>0$ such that diameter of holes of $U$ are lower bounded by $\sigma$. Since $\lim _{n \rightarrow \infty}\left|a_{n}-b_{n}\right|=0$, there exists $N \in \mathbb{N}$ such that $\left|a_{n}-b_{n}\right|<\sigma \forall n \geq N$. Then, we see that we have $\left|\frac{x-b_{n}}{x-a_{n}}\right|=1 \forall x \in U$, $\forall n \geq N$. Therefore, for $m \geq N$ we have

$$
\left\|h_{m+1}-h_{m}\right\|_{U}=\left\|h_{N}\right\|_{U}\left\|\frac{x-b_{m+1}}{x-a_{m+1}}-1\right\|_{U} .
$$

So, as $h_{N}$ is trivially bounded on $U$ and as

$$
\left\|\frac{x-b_{m+1}}{x-a_{m+1}}-1\right\|_{U}=\left\|\frac{a_{m+1}-b_{m+1}}{x-a_{m+1}}\right\|_{U} \leq \frac{\left|a_{m+1}-b_{m+1}\right|}{\sigma}
$$

we see that $\lim _{m \rightarrow \infty}\left\|h_{m+1}-h_{m}\right\|_{U}=0$. Therefore $h=\prod_{n=0}^{\infty}\left(\frac{x-b_{n}}{x-a_{n}}\right)$ is an analytic element on any $D$-admissible set $U$ and consequently $h \in \mathcal{A}(D)$. 
Similarly we show that $l=\prod_{n=0}^{\infty}\left(\frac{x-\alpha_{n}}{x-\beta_{n}}\right)$ is an element of $\mathcal{A}(D)$. As $g=h l, g$ is an element of the $K$-algebra $\mathcal{A}(D)$. But clearly, $g \notin H(D)$. Finally we check that $\frac{g^{\prime}}{g}=f$. By Theorem 29, the space of solutions $\mathcal{S}(f)$ has dimension 1 and then it is the subspace generated by $g$ in $\mathcal{A}(D)$.

\section{References}

1. Y. Amice, Les nombres p-adiques, P.U.F., Paris (1975).

2. V. G. Berkovich, "Spectral theory and analytic geometry over non archimedean fields," Mathematical Surveys and Monographs 33, Amer. Math. Soc., Providence, RI, 1990.

3. S. Bosch, U. Grüntzer and R. Remmert, "Non-Archimedean Analysis," Springer-Verlag, Berlin, Heidelberg, New York, 1984.

4. A. Escassut, Éléments analytiques et filtres percés sur un ensemble infraconnexe, Ann. Mat. Pura Appl. (4) 110 (1976), 335-352.

5. A. Escassut, $T$-filtres, ensembles analytiques et transformation de Fourier p-adique, Ann. Inst. Fourier (Grenoble) 25(2) (1975), 45-80.

6. A. Escassut, "Analytic elements in p-adic analysis," World Scientific Publishing, Singapore, 1995.

7. J. Fresnel And M. VAn-Der Put, "Géométrie analytique rigide et applications," Birkhäuser, Boston-Basel-Stuttgart, 1981.

8. G. Garandel, Les semi-normes multiplicatives sur les algèbres d'éléments analytiques au sens de Krasner, Indag. Math. 37(4) (1975), 327-341.

9. B. Guennebaud, Sur une notion de spectre pour les algèbres normées ultramétriques, Thèse, Université de Poitiers (1973).

10. R. Karlowski and P. Ullrich, A Tate-theoretic view of Krasner's non-archimedean function theory, Math. Ann. 289 (1991), 403-419.

11. M. Krasner, Prolongement analytique uniforme et multiforme dans les corps valués complets. Les tendances géométriques en algèbre et théorie des nombres, Clermont-Ferrand (1964), 94-141. Centre National de la Recherche Scientifique, 1966, (Colloques internationaux de C.N.R.S. Paris, 143).

12. N. MainetTi, Algebras of abstract analytic elements, in " $p$-adic functional analysis," Lecture Notes in Pure and Applied Mathematics 192, Dekker, New York, 1997, pp. 281-295. 
13. Y. Morita, Analytic functions on an open subset of $\mathbb{P}^{1}(k)$, J. Reine Angew. Math. 311/312 (1979), 361-383.

14. PH. RoBBA, Fonctions analytiques sur les corps valués ultramétriques complets. Prolongement analytique et algèbres de Banach ultramétriques, Astérisque 10 (1973), 109-220.

15. M. C. Sarmant And A. Escassut, T-suites idempotentes, Bull. Sci. Math. (2) 106(3) (1982), 289-303.

16. M. C. SARmant And A. Escassut, The equation $y^{\prime}=\omega y$ and meromorphic products, in " $p$-adic functional analysis," Lecture Notes in Pure and Applied Math. 137, Dekker, New York, 1992, pp. $157-175$.

17. J. TATE, Rigid analytic spaces, Invent. Math. 12 (1971), 257-289.

\author{
Laboratoire de Mathématiques Pures \\ Université Blaise Pascal (Clermont-Ferrand) \\ Complexe Scientifique des Cézeaux \\ 63177 Aubiere Cedex \\ FRANCE \\ e-mail: boussaf@ucfma.univ-bpclermont.fr
}

Primera versió rebuda el 29 de gener de 1998, darrera versió rebuda el 19 de juny de 1998 